\title{
Evolution of elementary excitations at a doped polar semiconductor surface in a depletion-layer formation process
}

\author{
Takeshi Inaoka \\ Department of Materials Science and Technology, Faculty of Engineering, Iwate University, 4-3-5 Ueda, Morioka, \\ Iwate 020-8551, Japan
}

(Received 3 May 2000; revised manuscript received 22 December 2000; published 5 April 2001)

\begin{abstract}
We investigate the evolution of elementary excitations at a doped polar semiconductor surface in a depletion-layer formation process. The elementary excitations analyzed are two coupled plasmon-phonon modes and a surface optical-phonon mode involving screening charges. Surface excitations of free carriers are calculated by taking account of the Coulomb interaction between carriers, the dynamical exchange-correlation effect, and the coupling with surface polar phonons, on the basis of thermal-equilibrium states calculated self-consistently by the local-density approximation. We focus our attention on the coupling character and the spatial structure of each surface-excitation mode, as well as the energy dispersion and the energy-loss intensity involved in the excitation. The induced charge-density distribution in the excitation mode is composed of a carrier component due to carrier density fluctuation, a phonon component arising from longitudinal polarphonon polarization, and two on-surface components originating from the termination of the polar phonon and background polarization at the surface. The coupling character is elucidated by the phase relation and the amplitude ratio among these induced charge components. The spatial structure is visualized by the contour map of the induced charge-density distribution. We follow the variation in the coupling character and the spatial structure in the depletion-layer formation process. Our analysis gives a clear explanation of the variation in each of the three loss peaks in the electron energy-loss spectrum.
\end{abstract}

DOI: 10.1103/PhysRevB.63.165322

PACS number(s): 73.20.Mf, 71.45.Gm, 71.38.-k

\section{INTRODUCTION}

Adsorption on a doped semiconductor surface often induces a gradual formation of a carrier-depletion or carrieraccumulation layer at the surface. Hydrogen adsorption on an $n$-type GaAs (110) surface is a typical case of the depletion-layer formation. As shown experimentally ${ }^{1-5}$ and theoretically, ${ }^{6-9}$ on a relaxed clean (110) surface of GaAs, the $\mathrm{Ga}$ and As atoms in the top layer are displaced toward the inside and into the vacuum, respectively, and the neighboring $\mathrm{Ga}$ and As surface atoms form a relaxed dimer. This surface relaxation entails electron charge transfer from the Ga atom to the As atom in the dimer, ${ }^{4}$ as required by the electron-counting model. ${ }^{10,11}$ This model asserts that all As dangling bonds be terminated by electron lone pairs, while all Ga dangling bonds be empty. Accordingly, there occur no surface states in the band gap. However, when a hydrogen (H) atom bonds to one of the relaxed dimer, it operates to remove the relaxation of the dimer, which results in the appearance of a midgap surface state from the other atom of the dimer. The fact that $\mathrm{H}$ adsorption removes the surface relaxation is supported both experimentally ${ }^{12-18}$ and theoretically. ${ }^{19-24}$

The theoretical calculation for the case of submonolayer $\mathrm{H}$ coverages in Refs. 22-24 provides a clear picture of the above-stated derelaxation. As shown by the self-consistent pseudopotential method, there is just a little difference in binding energy between the $\mathrm{Ga}-\mathrm{H}$ bond and the As- $\mathrm{H}$ bond at the surface..$^{22,24}$ On the experimental side, by means of high-resolution electron energy-loss spectroscopy (HREELS), one can detect both the Ga-H and the As-H stretching vibrations in an energy region of $200-300 \mathrm{meV}$ even at the lowest exposures. ${ }^{25,26}$ These results indicate that $\mathrm{H}$ atoms can bond to both the $\mathrm{Ga}$ and As surface atoms even in the smallest coverage range. With the midgap surface states induced, some electrons in the conduction band drop into these surface states, which create a carrier-depletion layer. Therefore, hydrogen adsorption on an n-type GaAs (110) surface causes a gradual formation of a carrierdepletion layer at the surface with an increase in coverage. The $\mathrm{H}$ coverage becomes close to one monolayer only after $10^{4}$ langmuir (L) ${ }^{26}$ or so much exposure (see Table I in Ref. 18). The above formation of the depletion layer proceeds at much lower exposures, namely, at coverages much smaller than one monolayer.

In contrast to the clean (110) surface where the surface relaxation prevents the appearance of surface states, the clean GaAs (001) surface already has surface states in the band gap, which leads to the formation of a depletion layer. The molecular-beam-epitaxy (MBE) grown (001) surface of $n$-doped GaAs is reported to have a substantial depletion layer. $^{27,28}$ To prevent contamination and damage on the MBE-grown surface, Gray-Grychowski et al. ${ }^{27}$ employed a protective-overlayer technique, and Noguchi, Hirakawa and Ikoma $^{28}$ examined a pristine surface immediately after MBE growth without exposure to air. In view of these careful treatments, the above-mentioned surface states are considered to be intrinsic to the clean (001) surface. Furthermore, the clean (001) surface of $n$-type $\mathrm{InSb}$ is also reported to have a considerable depletion layer. ${ }^{29,30}$

The carrier density distribution and the effective oneelectron potential in the absence and the presence of a surface space-charge layer can be calculated self-consistently by several schemes. To perform self-consistent calculations with reasonable efforts, carrier charges in surface states are 
so often replaced by a uniform distribution of surface charges. One of these schemes is a Hartree calculation employing a parametrized Morse potential for the effective potential. ${ }^{31,32}$ This parameterized scheme facilitates selfconsistent calculations of semi-infinite systems. Another scheme is a complete Hartree calculation of a carrier system in a semiconductor film. ${ }^{33-35}$ In this scheme, the effective potential is calculated numerically without parametrizing it. One can obtain a self-consistent solution more easily by the film geometry than by the semi-infinite one. A recent one is a local-density-approximation (LDA) calculation of a carrier system in the semiconductor film. ${ }^{36}$ By this scheme, the present author has investigated the evolution of electron states at the surface in a depletion-layer formation process. In this calculation, the film thickness is taken to be so sufficiently large that the carrier-density distribution at the film surface becomes equivalent to that at the surface of the semiinfinite system, even in the presence of a thick depletion layer. The result of this calculation provides a numerical basis for the present work of calculating surface excitations quantitatively.

The existence of the depletion layer at the surface exerts a significant influence on surface excitations of carriers often coupled with surface polar phonons. By means of HREELS, one can observe these coupled surface-excitation modes at the $n$-doped GaAs (110) surface in the depletion-layer formation process induced by $\mathrm{H}$ adsorption. ${ }^{26,35,37}$ Particularly, in Ref. 35, the remarkable variation in the EEL spectrum with exposure to hydrogen clarifies the evolution of the coupled surface modes in the depletion-layer formation process. In these spectra, three loss peaks are clearly resolved because of the suitable choice of the doping level. The higher-energy and the lower-energy ones of the three peaks are attributed to the coupled carrier plasmon-polar phonon modes, and interior excitations below the depletion layer play the leading role in these modes. The intermediate peak is ascribed to the surface optical-phonon (Fuchs-Kliewer) mode that undergoes the screening effect due to the presence of carriers. The phonon polarization active between the surface and screening charges below plays the principal part in this mode.

At a lower or higher doping level, there appear only two loss peaks (and sometimes their multiple-loss peaks) in the EEL spectrum. At the lower doping level, ${ }^{37}$ the lower coupled mode considerably below the polar-phonon energy regime acquires plasmon character, and the upper coupled mode and the optical-phonon mode involving screening charges coalesce into a single loss peak. At the higher doping level, ${ }^{26}$ however, the upper coupled mode becomes plasmonlike, and the lower coupled mode has its weak intensity buried in the intermediate loss peak or in the tail of the quasielastic peak. In passing, surface excitations on the clean $n$-doped GaAs (001) or InSb (001) surface with its intrinsic surface states in Refs. 27-30 correspond to the latter case of the higher doping level.

In relation to EELS measurements, there have been several theoretical studies that are centered on calculating the surface energy-loss function. This function indicates the intensity of the energy loss involved in the dynamical response of the surface to the external potential. In Ref. 35, to analyze their experimental result, Chen et al. calculated EEL spectra by using a semiclassical local-response theory. ${ }^{38}$ At each stage of the formation process, they estimated the depletionlayer thickness by fitting calculated spectra with experimental ones. In some other theoretical studies, the random-phase approximation (RPA) is employed to take nonlocal effects into consideration. In Ref. 39, to examine the experimental result of the $n$-type GaAs (001) surface with a depletion layer, ${ }^{27}$ Inaoka and Chihara calculated EEL spectra by combining the RPA calculation with Schaich's scheme. ${ }^{40}$ They used a simplified model where carriers are confined by an infinite potential barrier at the interface between the semiinfinite system and a carrier-free layer placed on it. This calculation includes coupling of plasmons with polar phonons. In a more realistic treatment, the eigenfunctions in thermal equilibrium calculated self-consistently are employed in the RPA calculation of surface excitations. In Ref. 41, using the thermal-equilibrium states calculated by a parametrized Hartree scheme, Ehlers and Mills investigated the energy dispersion and the Landau damping in surface plasmons of semi-infinite systems in the presence and the absence of a depletion layer. They presented the energy dispersion for some carrier concentrations. Although they considered the coupling of plasmons with polar phonons in a part of Ref. 41, it is restricted only to the case of no depletion layer. In Refs. 42 and 43, assuming a semiconductor film with and without a depletion layer at each surface, Streight and Mills analyzed the energy dispersion of surface plasmons, and the resonance feature of the surface energy-loss function along the plasmon-dispersion branch. In this RPA calculation, they employed the numerical result of a complete Hartree calculation of the thermal-equilibrium states. Because of a film geometry, surface plasmons on both sides of the film interact considerably with each other. Their analysis takes no account of the coupling of plasmons with polar phonons. Incidentally, Refs. 41 and 43 mentioned above also include an analysis of surface plasmons in the presence of a carrier-accumulation layer.

In the present paper, we examine the evolution of the two coupled plasmon-phonon modes at the surface and the surface optical-phonon mode involving screening charges in a depletion-layer formation process. These surface excitations are calculated by means of the time-dependent LDA scheme on the basis of the numerical result of the thermalequilibrium states obtained from the LDA calculation in Ref. 36. We pay special attention to the spatial structure and the coupling character of the surface excitations, as we did in Refs. 44 and 45 for the surface without a depletion layer and with a flat band assumed. The spatial structure can be visualized in the contour map of the induced charge-density distribution. The coupling character can be clarified by decomposing the induced charge-density distribution. The induced charge density below the surface can be resolved into the carrier component due to carrier density fluctuation and the phonon component arising from longitudinal polar-phonon polarization. The induced surface-charge density originates from the termination of the polar-phonon and background polarization at the surface, and so it can be decomposed into 
the phonon component and the background one. The coupling character shows itself in the phase relation and the amplitude ratio among these components in spatial variation along the propagating direction. We analyze the variation in the spatial structure and the coupling character in the depletion-layer formation process. In passing, a preliminary report of the present paper is already presented in Ref. 46 .

\section{THEORY}

In this section, we describe a theoretical framework for the following analysis. Our carrier system is assumed to be in a uniform background that is electrically positive owing to ionized donors and whose polarization is described by a dielectric function. In $n$-type polar semiconductors with large effective Bohr radii, such as $n$-GaAs, $n$-InAs, and $n$-InSb, increasing the doping level readily leads to such a high effective density of carriers that an impurity band due to donors merges into the conduction band. In this case, ionized donors can be spread out into a uniform distribution of positive charges. In the presence of the surface, the carrier density falls and vanishes at the surface. The characteristic length to describe this density fall near the surface (several tens of $\AA$ or $\sim 100 \AA$ ) is much longer than that needed to describe the penetration of electronic states into the vacuum $(\sim \AA)$. Accordingly, we can assume an infinite potential barrier at the background surface, and can impose a boundary condition that envelope functions of carriers vanish there. When some carriers in the conduction band fall into surface states in the band gap, the surface becomes negatively charged, and a carrier-depletion layer is formed right on the inside of this negatively charged surface. We describe these carriers in surface states by a uniform distribution of surface charges that extends just outside and along the background surface. $^{31-36}$

We calculate the dynamical response of the surface with or without a depletion layer to a periodic and oscillatory external potential that has surface-parallel wave vector $\mathbf{Q}$ and angular frequency $\omega$. This dynamical response involves the coupling of surface excitations of carriers with surface polar phonons. The $z$ axis is taken to be normal to the surface so that the semi-infinite material extends in the region $z<0$. When the external potential $U$ is produced by external charges outside the material, this potential penetrates into the material like $e^{Q z}$. The dynamical response of the surface can be described by the following pair of equations:

$$
\begin{aligned}
& V(z)=V(0) e^{Q z} \\
& +\frac{1}{\varepsilon_{\mathrm{PH}}(\omega)} \frac{2 \pi}{Q}\left[\int_{-\infty}^{0} d z^{\prime} e^{-Q\left|z^{\prime}-z\right|} \delta \rho_{\mathrm{EL}}\left(z^{\prime}\right)\right. \\
& \left.-e^{Q z} \int_{-\infty}^{0} d z^{\prime} e^{Q z^{\prime}} \delta \rho_{\mathrm{EL}}\left(z^{\prime}\right)\right]+\frac{\partial V_{\mathrm{XC}}\left[\rho_{\mathrm{EL}}(z)\right]}{\partial \rho_{\mathrm{EL}}} \delta \rho_{\mathrm{EL}}(z) \\
& \delta \rho_{\mathrm{EL}}(z)=\int_{-\infty}^{0} d z^{\prime} \chi\left(z, z^{\prime}\right) V\left(z^{\prime}\right) .
\end{aligned}
$$

In these equations, $\delta \rho_{\mathrm{EL}}$ and $V$ denote the induced charge density due to carrier density fluctuation and the selfconsistent total potential, respectively. Complete forms $V(\mathbf{Q}, z, \omega), \delta \rho_{\mathrm{EL}}(\mathbf{Q}, z, \omega)$, and $\chi\left(\mathbf{Q}, z, z^{\prime}, \omega\right)$ are abbreviated as $V(z), \delta \rho_{\mathrm{EL}}(z)$, and $\chi\left(z, z^{\prime}\right)$, respectively. The second and fourth terms on the right-hand side (rhs) of Eq. (1) represent the induced Coulomb potential due to $\delta \rho_{\mathrm{EL}}$ and the dynamical exchange-correlation (XC) contribution in the LDA, respectively. The sum of the first and third terms on the rhs of Eq. (1) with a common factor $e^{Q z}$ is made up of the external potential and the induced potential due to the termination of the polar-phonon and background polarization at $z=0$. By means of the Lorentzian oscillator model, the polar-phonon and background polarization is incorporated into this equation in the form of the dielectric function

$$
\varepsilon_{\mathrm{PH}}(\omega)=\varepsilon_{\infty}+\frac{\left(\varepsilon_{0}-\varepsilon_{\infty}\right) \omega_{\mathrm{TO}}^{2}}{\omega_{\mathrm{TO}}{ }^{2}-\omega^{2}-i \gamma \omega} .
$$

In this equation, $\omega_{\mathrm{TO}}$ is the transverse optical-phonon frequency of long wavelength, $\varepsilon_{\infty}$ and $\varepsilon_{0}$ are the highfrequency and static dielectric constants, respectively, and $\gamma$ is the phonon relaxation-rate constant. This local-response scheme gives a good description of our excitation modes where the spatial variation is slow on the scale of lattice constants. In the XC term, $\partial V_{\mathrm{XC}}\left[\rho_{\mathrm{EL}}(z)\right] / \partial \rho_{\mathrm{EL}}$ denotes the density derivative of the $\mathrm{XC}$ potential in thermal equilibrium, ${ }^{47}$ where $\rho_{\mathrm{EL}}(z)$ is the carrier charge density at $z$ in thermal equilibrium. For the exchange part in $V_{\mathrm{XC}}$, we employ a finite-temperature formula in Ref. 48 with the Rydberg unit replaced by the effective Rydberg unit. The explicit form is given by Eq. (9) in Ref. 36. For the correlation part in $V_{\mathrm{XC}}$, we adopt a zero-temperature formula in Ref. 49, because there seems to be no handy formula that allows for temperature effects, and our carrier system is relatively well degenerate. This correlation formula produces no artificial discontinuity in its density derivative, because it is expressed by a single analytic function up to such a high effective density that the effective density parameter $r_{s}^{*}$ becomes less than unity. Here, the parameter $r_{s}^{*}$ is defined by $(4 / 3) \pi\left(r_{s}^{*} a_{B}^{*}\right)^{3} n=1$ with the effective Bohr radius $a_{B}^{*}$ and the carrier density $n$. The familiar formula in Ref. 50 leads to discontinuity at $r_{s}^{*}=1$, because two different analytic forms are employed for a lower-density range with $r_{s}^{*}>1$ and a higher one with $r_{s}^{*}<1$. Equation (2) implies that the induced charge density $\delta \rho_{\mathrm{EL}}$ arises from the response of our carrier system to the total potential $V$. The susceptibility $\chi$ is expressed as ${ }^{51}$

$$
\begin{aligned}
& \chi\left(z, z^{\prime}\right) \\
& =2 e^{2} \sum_{\nu, \nu^{\prime}} \int \frac{d^{2} \mathbf{K}}{(2 \pi)^{2}} \frac{f\left(\mathbf{K}+\mathbf{Q}, \nu^{\prime}\right)-f(\mathbf{K}, \nu)}{E\left(\mathbf{K}+\mathbf{Q}, \nu^{\prime}\right)-E(\mathbf{K}, \nu)+\hbar \omega+i \eta} \\
& \quad \times \zeta_{\nu}{ }^{*}\left(z^{\prime}\right) \zeta_{\nu^{\prime}}\left(z^{\prime}\right) \zeta_{\nu^{\prime}} *(z) \zeta_{\nu}(z)
\end{aligned}
$$

Each eigenstate is specified by a surface-parallel wave vector $\mathbf{K}$ and a quantum number $\nu$ for surface-normal motion of 
carriers. The symbols $E(\mathbf{K}, \nu)$ and $f(\mathbf{K}, \nu)$ signify the energy and the average occupation of eigenstate $(\mathbf{K}, \nu)$, respectively, and $E(\mathbf{K}, \nu)$ is expressed as

$$
E(\mathbf{K}, \nu)=\hbar^{2} \mathbf{K}^{2} / 2 m^{*}+E_{\nu},
$$

in terms of the effective mass $m^{*}$ and the surface-normal component $E_{\nu}$. The function $\zeta_{\nu}(z)$ is the surface-normal component of the eigenfunction for $E_{\nu}, \eta$ is a very small positive constant, and $e(>0)$ is an absolute value of the electronic charge. In Ref. 36, using the LDA, the thermalequilibrium states have been calculated for a value of the depleted carrier density given at each stage of the depletionlayer formation process. The depleted carrier density $N_{s}$ is defined by

$$
N_{s}=\int_{-\infty}^{0}\left[n_{0}-n(z)\right] d z,
$$

in terms of the donor density $n_{0}$ and the carrier-density distribution $n(z)\left(=-\rho_{\mathrm{EL}}(z) / e\right)$ in thermal equilibrium. Eigenfunctions $\zeta_{\nu}(z)$ and eigenvalues $E_{\nu}$ are obtained in this calculation. Eigenfunctions $\zeta_{\nu}(z)$ and eigenvalues $E_{\nu}$ are given by solutions of the Schrödinger equation with an effective potential $V_{\text {eff }}$ determined self-consistently:

$$
\left[-\frac{\hbar^{2}}{2 m^{*}} \frac{d^{2}}{d z^{2}}+V_{\mathrm{eff}}(z)\right] \zeta_{\nu}(z)=E_{\nu} \zeta_{\nu}(z),
$$

where we measure $V_{\text {eff }}$ from its value for $z \rightarrow-\infty$. The $\nu$ or $\nu^{\prime}$ sum in Eq. (4) is performed over bound states with $E_{\nu}$ $<0$, if present, and mobile states with $E_{\nu}>0$. To facilitate the summation over mobile states, we impose a boundary condition $\zeta_{\nu}(-L)=0$ with a quantization length $L$, and take the limit of $L \rightarrow \infty$ at the final stage. It is convenient to employ wave numbers $k$ defined by $\hbar^{2} k^{2} / 2 m^{*}=E_{\nu}$ instead of $\nu$ and to express $\zeta_{\nu}$ as $\zeta_{k}$. Following a manipulation in Ref. 31 , we can convert the $\nu$ sum over mobile states into a $k$ integral. For each eigenfunction $\zeta_{k}(z)$, we define a function $\widetilde{\zeta}_{k}(z)$ that is proportional to $\zeta_{k}(z)$ and which varies as $\sqrt{ } 2 \sin [k z-\alpha(k)]$ for $z \rightarrow-\infty$. We take such a large distance $D$ that $\widetilde{\zeta}_{k}(z)$ becomes substantially equal to $\sqrt{2} \sin [k z$ $-\alpha(k)]$ in a region $z \leqslant-D$, and simultaneously let the length $L(\gg D)$ be sufficiently large so that $\widetilde{\zeta}_{k}(z)$ can be treated as a smooth function of $k$ in a range $-D \leqslant z \leqslant 0$. Here, we consider Eq. (7) with $\zeta_{\nu}$ and $E_{\nu}$ replaced by $\widetilde{\zeta}_{k}$ and $\hbar^{2} k^{2} / 2 m^{*}$, respectively. By subtracting Eq. (7) multiplied by $\partial \widetilde{\zeta}_{k}(z) / \partial k$ from the $k$ derivative of Eq. (7) multiplied by $\widetilde{\zeta}_{k}(z)$ and by integrating the resulting equation in $z$ from $z=-D$ to $z$ $=0$, we can obtain the relation:

$$
\begin{aligned}
\int_{-D}^{0} \widetilde{\zeta}_{k}^{2}(z) d z= & \frac{1}{2 k} \int_{-D}^{0} d z\left[\left\{\frac{\partial}{\partial k} \widetilde{\zeta}_{k}(z)\right\}\left\{\frac{\partial^{2}}{\partial z^{2}} \widetilde{\zeta}_{k}(z)\right\}\right. \\
& \left.-\widetilde{\zeta}_{k}(z) \frac{\partial}{\partial k} \frac{\partial^{2}}{\partial z^{2}} \widetilde{\zeta}_{k}(z)\right] \\
= & D+\frac{d \alpha}{d k}-\frac{\sin [2(k D+\alpha)]}{2 k},
\end{aligned}
$$

which gives the integral value

$$
\int_{-L}^{0} \widetilde{\zeta}_{k}^{2}(z) d z=\int_{-L}^{-D} \widetilde{\zeta}_{k}^{2}(z) d z+\int_{-D}^{0} \widetilde{\zeta}_{k}^{2}(z) d z=L+\frac{d \alpha}{d k} .
$$

By recalling that $\zeta_{k}(z)$ is normalized by its integral over a range $-L \leqslant z \leqslant 0$, we find the relation

$$
\widetilde{\zeta}_{k}(z)=\left(L+\frac{d \alpha}{d k}\right)^{1 / 2} \zeta_{k}(z)
$$

On the other hand, from the boundary condition at $z=-L$, we obtain the spacing $\delta k$ between neighboring allowed $k$ values as

$$
\delta k=\frac{\pi}{L+d \alpha / d k} .
$$

The summation over mobile states $k$ in Eq. (4) takes the form

$$
\chi_{\mathrm{mob}}\left(z, z^{\prime}\right)=\sum_{k} A_{k}\left(z, z^{\prime}\right) \zeta_{k}(z) \zeta_{k}\left(z^{\prime}\right)
$$

which changes into a $k$ integral

$$
\chi_{\mathrm{mob}}\left(z, z^{\prime}\right)=\frac{1}{\pi} \int_{0}^{\infty} d k\left(L+\frac{d \alpha}{d k}\right) A_{k}\left(z, z^{\prime}\right) \zeta_{k}(z) \zeta_{k}\left(z^{\prime}\right),
$$

with the aid of Eq. (11). In view of Eq. (10), Eq. (13) can be rewritten as

$$
\chi_{\mathrm{mob}}\left(z, z^{\prime}\right)=\frac{1}{\pi} \int_{0}^{\infty} d k A_{k}\left(z, z^{\prime}\right) \widetilde{\zeta}_{k}(z) \widetilde{\zeta}_{k}\left(z^{\prime}\right) .
$$

To introduce $\widetilde{\zeta}_{k}(z)$ is quite helpful in summing over mobile states in Eq. (4). For each surface mode specified by $\mathbf{Q}$ and $\omega$, we can obtain $V(z) / V(0)$ and $\delta \rho_{\mathrm{EL}}(z) / V(0)$ as functions of $z$ by solving the pair of Eqs. (1) and (2) self-consistently.

The dynamical response of the surface entails induced charges, which generate an induced Coulomb potential $\phi$. We let $V_{C}$ signify the sum of $U$ and $\phi$. If we define the surface dielectric function $\varepsilon_{S}(\mathbf{Q}, \omega)$ by ${ }^{52}$

$$
\varepsilon_{S}(\mathbf{Q}, \omega)=\frac{\varepsilon_{\mathrm{PH}}(\omega)}{Q V_{C}(0)}\left[\frac{\partial}{\partial z} V_{C}(z)\right]_{z=-0},
$$

the induced Coulomb potential $\phi$ outside the material $(z$ $>0$ ) can be related to the external potential $U$ by

$$
\phi(z)=-\frac{\varepsilon_{S}(\mathbf{Q}, \omega)-1}{\varepsilon_{S}(\mathbf{Q}, \omega)+1} U(0) e^{-Q z} .
$$

In Eqs. (15) and (16), the complete forms $V_{C}(\mathbf{Q}, z, \omega)$, $\phi(\mathbf{Q}, z, \omega)$, and $U(\mathbf{Q}, z, \omega)$ are contracted to $V_{C}(z), \phi(z)$, and $U(z)$, respectively. In deriving Eq. (16), we employ Eq. (15) and the continuity of the $z$ component of the electric displacement at $z=0$. The prefactor with $\varepsilon_{S}$ on the rhs of Eq. (16) is a familiar form in the image method of electrostatics. In terms of $\delta \rho_{\mathrm{EL}}(z)$, the surface dielectric function defined by Eq. (15) can be written as ${ }^{52}$ 


$$
\varepsilon_{S}(\mathbf{Q}, \omega)=\varepsilon_{\mathrm{PH}}(\omega)-\frac{4 \pi}{Q} \int_{-\infty}^{0} d z^{\prime} e^{Q z^{\prime}} \frac{\delta \rho_{\mathrm{EL}}\left(z^{\prime}\right)}{V(0)}
$$

Here, we note that $V$ becomes equal to $V_{C}$ at the surface $z$ $=0$ where $\delta \rho_{\mathrm{EL}}$ vanishes. Surface excitations involve the energy loss that is equivalent to the work performed by external charges against the induced Coulomb potential. The energy loss per unit time and unit surface area is expressed as

$$
W=(Q \omega / \pi)|U(0)|^{2} \operatorname{Im}[-\phi(0) / U(0)],
$$

where Im denotes the imaginary part. In view of this expression, we define the surface energy-loss function $F(\mathbf{Q}, \omega)$ by

$$
F(\mathbf{Q}, \omega)=\operatorname{Im}[-\phi(0) / U(0)]=\operatorname{Im}[-V(0) / U(0)],
$$

which can be rewritten as

$$
F(\mathbf{Q}, \omega)=\operatorname{Im}\left[-2 /\left\{\varepsilon_{S}(\mathbf{Q}, \omega)+1\right\}\right],
$$

with the aid of Eq. (16).

The induced charge density $\delta \rho$ below the surface $(z<0)$ is composed of the carrier component $\delta \rho_{\mathrm{EL}}$ due to carrier density fluctuation and the phonon component $\delta \rho_{\mathrm{PH}}$ arising from longitudinal polar-phonon polarization:

$$
\delta \rho=\delta \rho_{\mathrm{EL}}+\delta \rho_{\mathrm{PH}} .
$$

On the basis of the Lorentzian oscillator model, the polarphonon polarization $\mathbf{P}(\mathbf{r}, \omega)$ is given by

$$
\left(\omega_{\mathrm{TO}}{ }^{2}-\omega^{2}-i \gamma \omega\right) \mathbf{P}(\mathbf{r}, \omega)=-\frac{\varepsilon_{0}-\varepsilon_{\infty}}{4 \pi} \omega_{\mathrm{TO}}^{2} \operatorname{grad} V_{C}(\mathbf{r}, \omega),
$$

and this polarization yields the induced charge density

$$
\delta \rho_{\mathrm{PH}}(\mathbf{r}, \omega)=-\operatorname{div} \mathbf{P}(\mathbf{r}, \omega)=\frac{\varepsilon_{\mathrm{PH}}(\omega)-\varepsilon_{\infty}}{4 \pi} \Delta V_{C}(\mathbf{r}, \omega) .
$$

By combining this equation with Poisson's equation

$$
-\varepsilon_{\mathrm{PH}}(\omega) \Delta V_{C}(\mathbf{r}, \omega)=4 \pi \delta \rho_{\mathrm{EL}}(\mathbf{r}, \omega),
$$

and by adopting partially Fourier-transformed representation, we can obtain the relation between $\delta \rho_{\mathrm{EL}}$ and $\delta \rho_{\mathrm{PH}}$ as

$$
\delta \rho_{\mathrm{PH}}(\mathbf{Q}, z, \omega)=-\frac{\varepsilon_{\mathrm{PH}}(\omega)-\varepsilon_{\infty}}{\varepsilon_{\mathrm{PH}}(\omega)} \delta \rho_{\mathrm{EL}}(\mathbf{Q}, z, \omega) .
$$

Termination of the polarization at the surface $z=0$ produces the induced surface-charge density $\sigma$, which consists of the component $\sigma_{\mathrm{PH}}$ due to the polar-phonon polarization and the component $\sigma_{\mathrm{BG}}$ owing to the background polarization described by $\varepsilon_{\infty}$. These two components are expressed as

$$
\begin{aligned}
\sigma_{\mathrm{PH}}(\mathbf{Q}, \omega) & =-\frac{\varepsilon_{\mathrm{PH}}(\omega)-\varepsilon_{\infty}}{4 \pi}\left[\frac{\partial}{\partial z} V_{C}(z)\right]_{z=-0} \\
& =-\frac{Q}{4 \pi} \frac{\varepsilon_{\mathrm{PH}}(\omega)-\varepsilon_{\infty}}{\varepsilon_{\mathrm{PH}}(\omega)} \varepsilon_{S}(\mathbf{Q}, \omega) V(0),
\end{aligned}
$$

and

$$
\begin{aligned}
\sigma_{\mathrm{BG}}(\mathbf{Q}, \omega) & =-\frac{\varepsilon_{\infty}-1}{4 \pi}\left[\frac{\partial}{\partial z} V_{C}(z)\right]_{z=-0} \\
& =-\frac{Q}{4 \pi} \frac{\varepsilon_{\infty}-1}{\varepsilon_{\mathrm{PH}}(\omega)} \varepsilon_{S}(\mathbf{Q}, \omega) V(0),
\end{aligned}
$$

where definition of $\varepsilon_{S}(\mathbf{Q}, \omega)$ in Eq. (15) is used. These two components are related to each other by

$$
\sigma_{\mathrm{PH}}(\mathbf{Q}, \omega)=\frac{\varepsilon_{\mathrm{PH}}(\omega)-\varepsilon_{\infty}}{\varepsilon_{\infty}-1} \sigma_{\mathrm{BG}}(\mathbf{Q}, \omega) .
$$

The coupling character in each surface mode can be elucidated by the phase relation and the amplitude ratio of $\delta \rho_{\mathrm{EL}}$ and $\delta \rho_{\mathrm{PH}}$ and of $\sigma_{\mathrm{BG}}$ and $\sigma_{\mathrm{PH}}$. The induced Coulomb potential $\phi(0)$ in Eq. (19) can be decomposed into contributions of $\delta \rho_{\mathrm{EL}}, \delta \rho_{\mathrm{PH}}, \sigma_{\mathrm{PH}}$, and $\sigma_{\mathrm{BG}}$ :

$$
\phi(0)=\phi_{\mathrm{EL}}(0)+\phi_{\mathrm{PH}}(0)+\phi_{\mathrm{SPH}}(0)+\phi_{\mathrm{SBG}}(0) .
$$

The first two components $\phi_{\mathrm{EL}}(0)$ and $\phi_{\mathrm{PH}}(0)$ due to $\delta \rho_{\mathrm{EL}}$ and $\delta \rho_{\mathrm{PH}}$, respectively, are expressed as

$$
\phi_{\mathrm{EL}}(0)=\frac{1}{\varepsilon_{\infty}} \frac{2 \pi}{Q} \int_{-\infty}^{0} d z^{\prime} e^{Q z^{\prime}} \delta \rho_{\mathrm{EL}}\left(z^{\prime}\right),
$$

and

$$
\phi_{\mathrm{PH}}(0)=\frac{1}{\varepsilon_{\infty}} \frac{2 \pi}{Q} \int_{-\infty}^{0} d z^{\prime} e^{Q z^{\prime}} \delta \rho_{\mathrm{PH}}\left(z^{\prime}\right) .
$$

The other components $\phi_{\mathrm{SPH}}(0)$ and $\phi_{\mathrm{SBG}}(0)$ generated by $\sigma_{\mathrm{PH}}$ and $\sigma_{\mathrm{BG}}$, respectively, are written as

$$
\phi_{\mathrm{SPH}}(0)=(2 \pi / Q) \sigma_{\mathrm{PH}}(\mathbf{Q}, \omega),
$$

and

$$
\phi_{\mathrm{SBG}}(0)=(2 \pi / Q) \sigma_{\mathrm{BG}}(\mathbf{Q}, \omega) .
$$

By substituting Eq. (29) into Eq. (19), we can resolve the energy-loss intensity into contributions of $\delta \rho_{\mathrm{EL}}, \delta \rho_{\mathrm{PH}}, \sigma_{\mathrm{PH}}$, and $\sigma_{\mathrm{BG}}$.

If we have the $x$ axis oriented to the direction of $\mathbf{Q}$, we can build up the spatial distribution of $\delta \rho$ and $\sigma$ in a surface mode of $\mathbf{Q}$ and $\omega$ as

$$
\delta \rho(x, z)=\delta \rho(\mathbf{Q}, z, \omega) e^{i Q x}+\text { c.c. }
$$

and

$$
\sigma(x)=\sigma(\mathbf{Q}, \omega) e^{i Q x}+\text { c.c. },
$$

respectively. Here, the symbol c.c. designates the complexconjugate term. The spatial structure of each surface mode can be visualized by the contour map of $\delta \rho(x, z)$ and the variation in $\sigma(x)$.

In $n$-type polar semiconductors such as $n$-GaAs, $n$-InAs, and $n$-InSb, the conduction-band dispersion is appreciably or highly nonparabolic, though it is almost isotropic. This nonparabolicity has a significant influence on excitations of carriers. Excitations in the conduction band are made up of 
electronic transitions around the Fermi level $\mu$, if the momentum transfer in these transitions is not so large. Therefore, we should use the effective mass $m^{*}$ determined from the energy dispersion around $\mu$. Here, we consider the dynamical response of a carrier system in the bulk to a periodic and oscillatory external potential with wave vector $\mathbf{q}$ and angular frequency $\omega$. The induced charge density $\delta \rho_{\mathrm{EL}}(\mathbf{q}, \omega)$ arises from the dynamical response of the carrier system to the total potential $V(\mathbf{q}, \omega)$, which is constituted of the external potential and the induced potential:

$$
\delta \rho_{\mathrm{EL}}(\mathbf{q}, \omega)=\chi(\mathbf{q}, \omega) V(\mathbf{q}, \omega) .
$$

The susceptibility $\chi(\mathbf{q}, \omega)$ is expressed as ${ }^{53}$

$$
\chi(\mathbf{q}, \omega)=2 e^{2} \int \frac{d^{3} \mathbf{k}}{(2 \pi)^{3}} \frac{f(k)-f(|\mathbf{k}+\mathbf{q}|)}{E(k)-E(|\mathbf{k}+\mathbf{q}|)+\hbar \omega+i \eta},
$$

where $E(k)$ is a nonparabolic but isotropic dispersion of the conduction band, and $f(k)$ and $\eta$ denote the Fermi-Dirac distribution function and a small positive constant, respectively. In the long-wavelength limit $(\mathbf{q} \rightarrow 0)$, Eq. (37) takes the same form as for a parabolic dispersion,

$$
\chi(\mathbf{q}, \omega) \approx \frac{n_{0} e^{2} \mathbf{q}^{2}}{m^{*} \omega^{2}},
$$

if the effective mass $m^{*}$ is defined by

$$
\frac{1}{m^{*}}=\frac{\beta}{3 \pi^{2} \hbar^{2} n_{0}} \int_{0}^{\infty} d k k^{2} f(k)[1-f(k)]\left[\frac{d E(k)}{d k}\right]^{2} .
$$

In Eq. (39), the symbol $\beta$ is defined by $\beta=1 / k_{B} T$ in terms of the Boltzmann constant $k_{B}$ and the absolute temperature $T$. The effective mass $m^{*}$ in Eq. (39) is determined by the energy dispersion around $\mu$, because the factor $f(k)[1$ $-f(k)$ ] becomes appreciable about a $k$ value corresponding to $\mu$. In the low-temperature limit $(T \rightarrow 0)$, Eq. (39) is reduced to the simple form ${ }^{54}$

$$
\frac{1}{m^{*}}=\frac{1}{\hbar^{2} k_{F}}\left[\frac{d E(k)}{d k}\right]_{k=k_{F}},
$$

where $k_{F}$ is the Fermi wave number. The energy dispersion $E(k)$ can be obtained by using a $\mathbf{k} \cdot \mathbf{p}$ method in Ref. 55. In this method, we can get $E(k)$ for each $k$ only by solving an algebraic equation [see Eq. (10) in Ref. 55]. By using $m^{*}$ defined by Eq. (39), we can incorporate the nonparabolicity effect into our parabolic calculational scheme, and can gain the correct energy of bulk carrier plasmon with $q=0$ corresponding to $n_{0}$. As mentioned in the following section, the energy of surface carrier plasmon with $Q=0$ is slightly lower than or almost equal to the above energy of the bulk carrier plasmon. Therefore, the effective mass in Eq. (39) also provides the accurate energy of the surface plasmon in the long-wavelength limit.

\section{RESULTS AND DISCUSSION}

By means of the theoretical framework in Sec. II, we examine the evolution of elementary excitations at a doped polar semiconductor surface in a depletion-layer formation process. As stated in Sec. I, hydrogen adsorption on an $n$-type GaAs (110) surface induces a gradual formation of a carrier-depletion layer at the surface. Our calculations deal with this case. The donor density $n_{0}$ and the temperature $T$ are taken to be $n_{0}=1.3 \times 10^{18} \mathrm{~cm}^{-3}$ and $T=300 \mathrm{~K}$, respectively, corresponding to the HREELS measurement in Ref. 35. Other parameter values are chosen to be $m * / m_{0}$ $=0.0702, \quad \varepsilon_{0}=12.85, \quad \varepsilon_{\infty}=10.88, \hbar \omega_{\mathrm{TO}}=33.25 \mathrm{meV}, \quad \eta$ $=0.05 \mathrm{meV}$, and $\hbar \gamma=0.05 \mathrm{meV}$, where $m_{0}$ denotes the freeelectron mass. As mentioned in Sec. II, the effective mass $m^{*}$ is determined by taking account of appreciable nonparabolicity of the conduction-band dispersion. The energy dispersion $E(k)$ in Eq. (39) has been calculated by using the band gap $E_{G}=1.423 \mathrm{eV}$, the spin-orbit splitting $\Delta_{\text {SO }}$ $=0.341 \mathrm{eV}$, and the band-edge effective mass $m_{0}^{*}$ $=0.0632 m_{0}$. The above values of $\varepsilon_{0}, \varepsilon_{\infty}, \hbar \omega_{\mathrm{TO}}, E_{G}, \Delta_{\mathrm{SO}}$ and $m_{0}^{*} / m_{0}$ are quoted from a review on GaAs of Ref. 56. By using the Lyddane-Sachs-Teller relation, we can obtain the longitudinal optical-phonon energy of long wavelength as $\hbar \omega_{\mathrm{LO}}=36.14 \mathrm{meV}$. The above values of $m^{*}$ and $\varepsilon_{0}$ give the effective Bohr radius $a_{B}^{*}=\varepsilon_{0} \hbar^{2} / m^{*} e^{2}=9.68 \mathrm{~nm}$, and the above carrier density $n_{0}$ corresponds to the effective density parameter $r_{s}^{*}=0.587$. This $r_{s}^{*}$ value smaller than unity indicates that our carrier system has a considerably high effective density. We employ considerably small values for relaxation-rate parameters $\eta$ and $\gamma$ so that we can readily find when the Landau damping begins to broaden the resonance peak in $\omega$ dependence of $F$.

As shown in a clear manner both experimentally ${ }^{35}$ and theoretically, ${ }^{44,45}$ there are three distinct coupled surface modes at each $Q$ in a finite and not too large $Q$ region. We investigate the energy-loss intensity, the energy dispersion, the coupling character, and the spatial structure of each of the three modes in the depletion-layer formation process.

\section{A. Higher-energy mode $A$}

This is the higher-energy one of the two coupled plasmon-phonon modes. Figure 1 shows the $\omega$ dependence of the surface energy-loss function $F$ at the surface-parallel wave number $Q=1 \times 10^{5} \mathrm{~cm}^{-1}$ (a), $3 \times 10^{5} \mathrm{~cm}^{-1}$ (b), and 5 $\times 10^{5} \mathrm{~cm}^{-1}$ (c). The numeral $0,1,2$, or 3 on each curve with calculated points on it indicates the depleted carrier density $N_{s}$ in units of $10^{12} \mathrm{~cm}^{-2}$. Each panel represents the variation in the resonance peak corresponding to the mode $A$ in the depletion-layer formation process. We should note that, in panel (c), the $F$ values for $N_{s}=0 \mathrm{~cm}^{-2}$ are scaled on the right ordinate, while those for the other $N_{s}$ values are graduated on the left ordinate. With formation of the depletion layer, the semi-infinite carrier system retreats from the surface into the depth, and surface excitations of this carrier system begin to suffer from the effect of the polarized depletion layer made of the dielectric medium. The mode energy shifts downward, and the energy-loss intensity declines with increase in $N_{s}$ at 

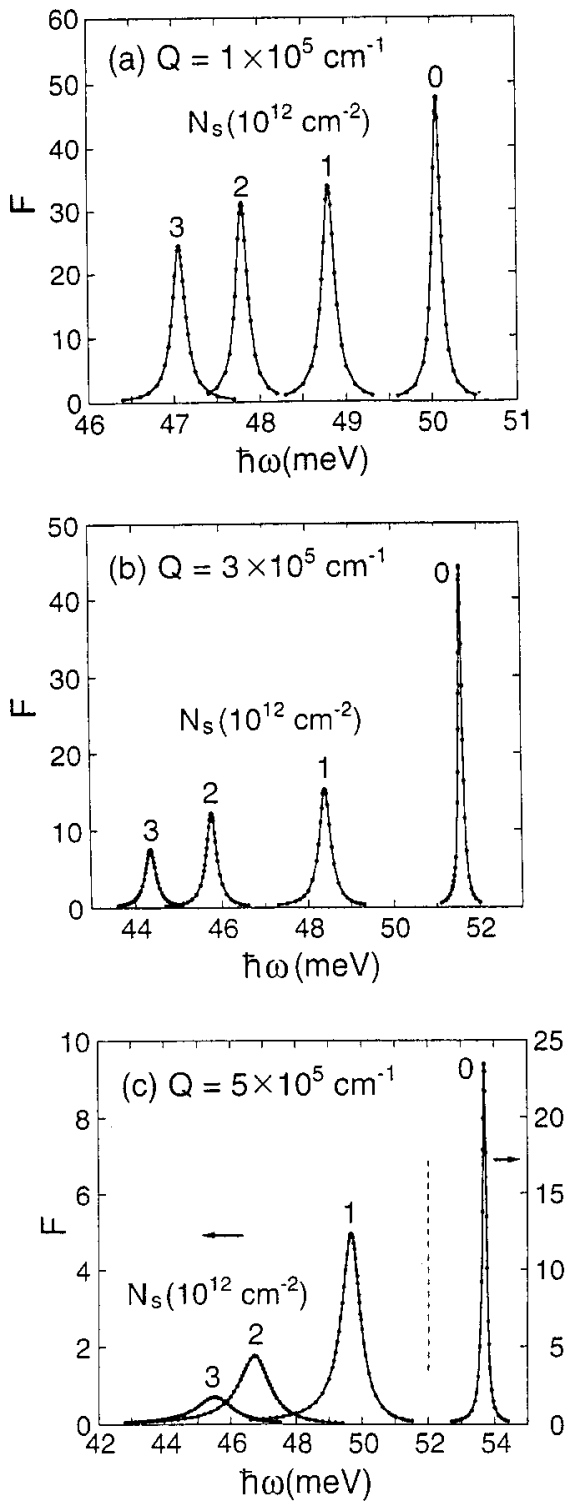

FIG. 1. $\omega$ dependence of the surface energy-loss function $F$ of the higher-energy mode $A$ at $Q=1 \times 10^{5} \mathrm{~cm}^{-1}$ (a), $3 \times 10^{5} \mathrm{~cm}^{-1}$ (b), and $5 \times 10^{5} \mathrm{~cm}^{-1}$. The numeral $0,1,2$, or 3 on each curve represents the depleted carrier density $N_{s}$ in units of $10^{12} \mathrm{~cm}^{-2}$. The curve for $N_{s}=0 \mathrm{~cm}^{-2}$ in (c) is scaled on the right ordinate.

each of the three $Q$ values. At $Q=1 \times 10^{5} \mathrm{~cm}^{-1}$, there is no substantial change in the peak width, and this width can be explained by the relaxation-rate constants $\eta$ and $\gamma$. At a larger $Q$ value, however, the resonance peak tends to broaden with increase in $N_{s}$. Especially, at $Q=5$ $\times 10^{5} \mathrm{~cm}^{-1}$, the resonance peak broadens and declines dramatically in the depletion-layer formation process. Our result can be compared with another result in Fig. 3 of Ref. 41, which exhibits the $N_{s}$ dependence of the resonance peak at $Q=8.6 \times 10^{5} \mathrm{~cm}^{-1}$ for $n_{0}=3 \times 10^{17} \mathrm{~cm}^{-3}$, and $T=300 \mathrm{~K}$. In the latter result for a larger $Q$ value, the resonance peak is considerably broad even in the absence of carrier depletion $\left(N_{s}=0 \mathrm{~cm}^{-2}\right)$. In view of this broadening at a larger $Q$ value, the resonance peak for $N_{s}=0 \mathrm{~cm}^{-2}$ in our result, which is very sharp over the whole $Q$ range analyzed here, is

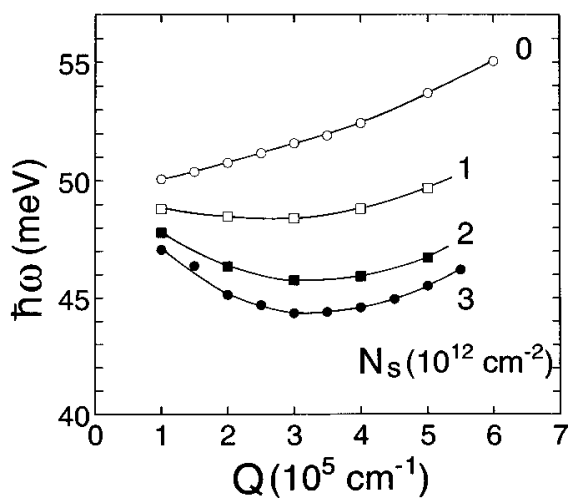

FIG. 2. Variation in the energy dispersion of the mode $A$ in the depletion-layer formation process. Each series of connected points shows the energy dispersion for the depleted carrier density $N_{s}$ specified in units of $10^{12} \mathrm{~cm}^{-2}$.

considered to become broader with further increase in $Q$. The above peak broadening can be ascribed to the Landau damping, namely, the effect of single-particle excitations on plasmons. The Landau damping begins to operate more effectively with a decrease in the mode energy, particularly at a larger $Q$ value.

We can obtain the energy dispersion by locating the peak position of $F$ at various $Q$ values. Figure 2 displays the variation in the energy dispersion in the depletion-layer formation process. Each connected series of calculated points indicates the energy dispersion for the depleted carrier density $N_{S}$ specified in units of $10^{12} \mathrm{~cm}^{-2}$. In our considerably high carrier concentration, the energy of the mode $A$ is significantly higher than the phonon energy regime, and as shown below, plasmon character becomes influential in this mode. In the absence of carrier depletion $\left(N_{s}=0 \mathrm{~cm}^{-2}\right)$, the mode energy shows an upward dispersion through the whole range of $Q$. With an increase in $N_{s}$, however, the dispersion curve descends, and the upward dispersion at $N_{s}=0 \mathrm{~cm}^{-2}$ evolves into a warped dispersion that is downward in a smaller $Q$ range and that turns upward in a larger $Q$ region. This warped dispersion is already obtained for surface plasmons in a slab system ${ }^{42,43}$ and for surface plasmons coupled with surface polar phonons in a semi-infinite system, ${ }^{39}$ both in the presence of a substantial depletion layer. In the above slab system, the surface plasmons on both sides interact with each other, which gives rise to two dispersion branches. The upper one of them corresponds to the dispersion branch in our result. The warped dispersion in the presence of the depletion layer results from competition between the plasmon nature that tends toward an upward dispersion and the effect of the depletion layer that leans toward a downward dispersion. The induced electric field generated by $\delta \rho$ attenuates in the depletion layer with a decay distance $\sim Q^{-1}$. We should notice that the depletion layer also has a large background dielectric constant $\varepsilon_{\infty}$. With an increase in $Q$, the induced electric field issuing from $\delta \rho$ and flowing through the depletion layer becomes more deeply embedded in the depletion layer of the dielectric medium, which leads to a downward dispersion. ${ }^{39}$ With an increase in $N_{s}$, the excitation mode at a larger $Q$ value shows a remarkable downward shift in en- 

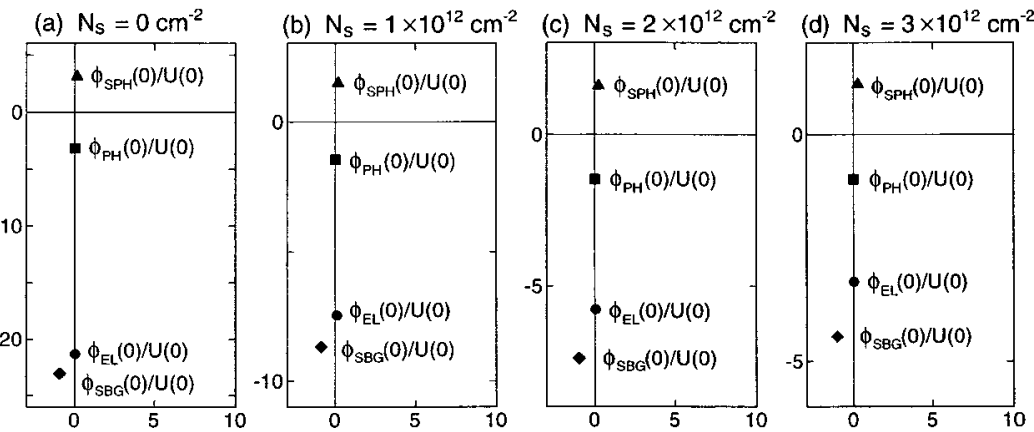

FIG. 3. Plot of $\phi_{\mathrm{EL}}(0) / U(0), \phi_{\mathrm{PH}}(0) / U(0)$, $\phi_{\mathrm{SBG}}(0) / U(0)$, and $\phi_{\mathrm{SPH}}(0) / U(0)$ of the mode $A$ at $Q=3 \times 10^{5} \mathrm{~cm}^{-1}$ for the depleted carrier density $N_{s}=0 \mathrm{~cm}^{-2}$ (a), $1 \times 10^{12} \mathrm{~cm}^{-2}$ (b), 2 $\times 10^{12} \mathrm{~cm}^{-2}(\mathrm{c})$, and $3 \times 10^{12} \mathrm{~cm}^{-2}(\mathrm{~d})$. In each panel, the abscissa and the ordinate represent the real and imaginary axes, respectively. This figure elucidates the phase relation and the amplitude ratio of the induced charge components. ergy, and simultaneously declines in energy-loss intensity significantly with the broadening of the resonance peak.

Here, we concentrate our attention on the mode $A$ at $Q$ $=3 \times 10^{5} \mathrm{~cm}^{-1}$, and investigate the coupling character and the spatial structure of this mode in the depletion-layer formation process. Figure 3 exhibits the plot of $\phi_{\mathrm{EL}}(0) / U(0)$, $\phi_{\mathrm{PH}}(0) / U(0), \phi_{\mathrm{SBG}}(0) / U(0)$, and $\phi_{\mathrm{SPH}}(0) / U(0)$ of this mode on the complex plane as a circle, a square, a diamond, and a triangle, respectively. The four panels (a)-(d) are assigned to the values of $N_{s}=0 \mathrm{~cm}^{-2}, 1 \times 10^{12} \mathrm{~cm}^{-2}, 2$ $\times 10^{12} \mathrm{~cm}^{-2}$, and $3 \times 10^{12} \mathrm{~cm}^{-2}$, respectively, and they correspond to the four points at $Q=3 \times 10^{5} \mathrm{~cm}^{-1}$ in Fig. 2. In each panel, the abscissa and the ordinate are the real and imaginary axes, respectively. The four complex values in each panel sum up to $\phi(0) / U(0)$, and the potential ratio $V(0) / U(0)\{=[U(0)+\phi(0)] / U(0)\}$ becomes almost pure imaginary at resonance, because the resonance corresponds to a pole of Eq. (20). The imaginary parts of $-\phi_{\mathrm{EL}}(0) / U(0),-\phi_{\mathrm{PH}}(0) / U(0),-\phi_{\mathrm{SBG}}(0) / U(0)$, and $-\phi_{\mathrm{SPH}}(0) / U(0)$ indicate the contributions of $\delta \rho_{\mathrm{EL}}, \delta \rho_{\mathrm{PH}}$, $\sigma_{\mathrm{BG}}$, and $\sigma_{\mathrm{PH}}$ to the surface energy-loss intensity $F$, respectively. These four imaginary parts add up to the value of $F$. This plot elucidates the phase relation and the amplitude ratio of the induced charge components in spatial variation along the direction of $\mathbf{Q}$, and the contribution of each induced charge component to $F$. Figure 4 displays the variation in the induced charge-density distribution in the depletionlayer formation process, along with the variation in the carrier-density profile in thermal equilibrium. The $x$ axis oriented to the direction of $\mathbf{Q}$ and the $z$ axis normal to the surface are scaled in units of $Q^{-1}(=33.3 \mathrm{~nm})$ and $a_{B}^{*}$ $(=9.68 \mathrm{~nm})$, respectively. In each panel with the $N_{s}$ value specified, the upper part exhibits the variation in $a_{B}^{*} \sigma(x) / V(0)$ (full curve, TL) and its decomposition into the background component (broken curve, BG) and the phonon component (dotted curve, PH). The left-lower portion is the contour map of $\left(a_{B}^{*}\right)^{2} \delta \rho(x, z) / V(0)$. As stated in Sec. II, the induced charge-density distribution $\delta \rho(x, z)$ is composed of the carrier component $\delta \rho_{\mathrm{EL}}(x, z)$ and the phonon component $\delta \rho_{\mathrm{PH}}(x, z)$. The right part shows the carrier-density distribution in thermal equilibrium with its $z$ scale matched to that in the contour map. The vertical broken line represents the homogeneous donor density. This density profile is adapted from Ref. 36.

Before analyzing surface excitations, we mention the carrier-density distribution in thermal equilibrium. As stated in Sec. II, we can assume an infinite potential barrier at the surface $z=0$, and impose a boundary condition that envelope functions of carriers vanish at the surface. In the absence of carrier depletion $\left(N_{s}=0 \mathrm{~cm}^{-2}\right)$, the carrier density $n$ rises quickly from zero, and a conspicuous peak emerges near the surface in the carrier-density profile, because carriers are confined by the surface potential barrier at $z=0$ [see the right part of Fig. 4(a)]. With an increase in thickness of the depletion layer, however, the above surface potential barrier reduces its influence on carriers, and the upward-bending effective potential starts to play the main role in restricting carriers to the inside. Therefore, the above peak in the density profile gradually dwindles away, and the carrier density begins to rise more slowly [follow the right parts of Figs. 4(a)-4(d)]. In view of considerable degeneracy of our carrier system, the rising rate of $n$ could be fairly well scaled by the inverse Fermi wave number $k_{F}^{-1}=2.96 \mathrm{~nm} \quad\left(k_{F}^{-1} / a_{B}^{*}\right.$ $=0.306)$ in the absence of carrier depletion and by the Thomas-Fermi screening length $k_{\mathrm{TF}}^{-1}=4.75 \mathrm{~nm}\left(k_{\mathrm{TF}}^{-1} / a_{B}^{*}\right.$ $=0.490$ ) in the presence of a substantial depletion layer.

Here, we investigate the coupling character and the spatial structure of the mode $A$ at $Q=3 \times 10^{5} \mathrm{~cm}^{-1}$. As seen from Fig. 3, regardless of $N_{s}$, the complex value $\phi_{\mathrm{EL}}(0) / U(0)$ has the same phase as $\phi_{\mathrm{PH}}(0) / U(0)$, and the former has a larger absolute value than the latter. This indicates that the variation in $\delta \rho_{\mathrm{EL}}(x, z)$ along $x$ is in a coherent-phase relation with that in $\delta \rho_{\mathrm{PH}}(x, z)$ along $x$, and that the variation in $\delta \rho_{\mathrm{EL}}(x, z)$ has a larger amplitude than that in $\delta \rho_{\mathrm{PH}}(x, z)$. This predominance of $\delta \rho_{\mathrm{EL}}$ over $\delta \rho_{\mathrm{PH}}$ in amplitude implies that, in our considerably high carrier concentration, plasmon character becomes influential in this mode. In contribution to the surface energy-loss intensity $F, \delta \rho_{\mathrm{EL}}$ and $\delta \rho_{\mathrm{PH}}$ cooperate to enhance $F$, because both $\phi_{\mathrm{EL}}(0) / U(0)$ and $\phi_{\mathrm{PH}}(0) / U(0)$ have negative imaginary parts. At $N_{s}=0 \mathrm{~cm}^{-2}$, the amplitude of $\delta \rho_{\mathrm{EL}}$ is much larger than that of $\delta \rho_{\mathrm{PH}}$. With an increase in $N_{s}$, however, the phonon component $\delta \rho_{\mathrm{PH}}$ increases its contribution, and its amplitude becomes as large as about one third of that of $\delta \rho_{\mathrm{EL}}$. Next, we turn our attention to $\phi_{\mathrm{SBG}}(0) / U(0)$ and $\phi_{\mathrm{SPH}}(0) / U(0)$. As seen from the phase relation and the amplitude ratio of these complex values, irrespective of $N_{s}$, the variation in $\sigma_{\mathrm{BG}}(x)$ is in an antiphase relation with that in $\sigma_{\mathrm{PH}}(x)$, and the background component $\sigma_{\mathrm{BG}}(x)$ has a larger amplitude. The imaginary parts of $\phi_{\mathrm{SBG}}(0) / U(0)$ and $\phi_{\mathrm{SPH}}(0) / U(0)$ are negative and positive, respectively. Accordingly, the background component $\sigma_{\mathrm{BG}}$ operates to intensify $F$, whereas $\sigma_{\mathrm{PH}}$ acts to suppress it. At $N_{s}=0 \mathrm{~cm}^{-2}$, the amplitude of $\sigma_{\mathrm{BG}}$ is much larger than that of $\sigma_{\mathrm{PH}}$. With an increase in $N_{s}$, however, the pho- 

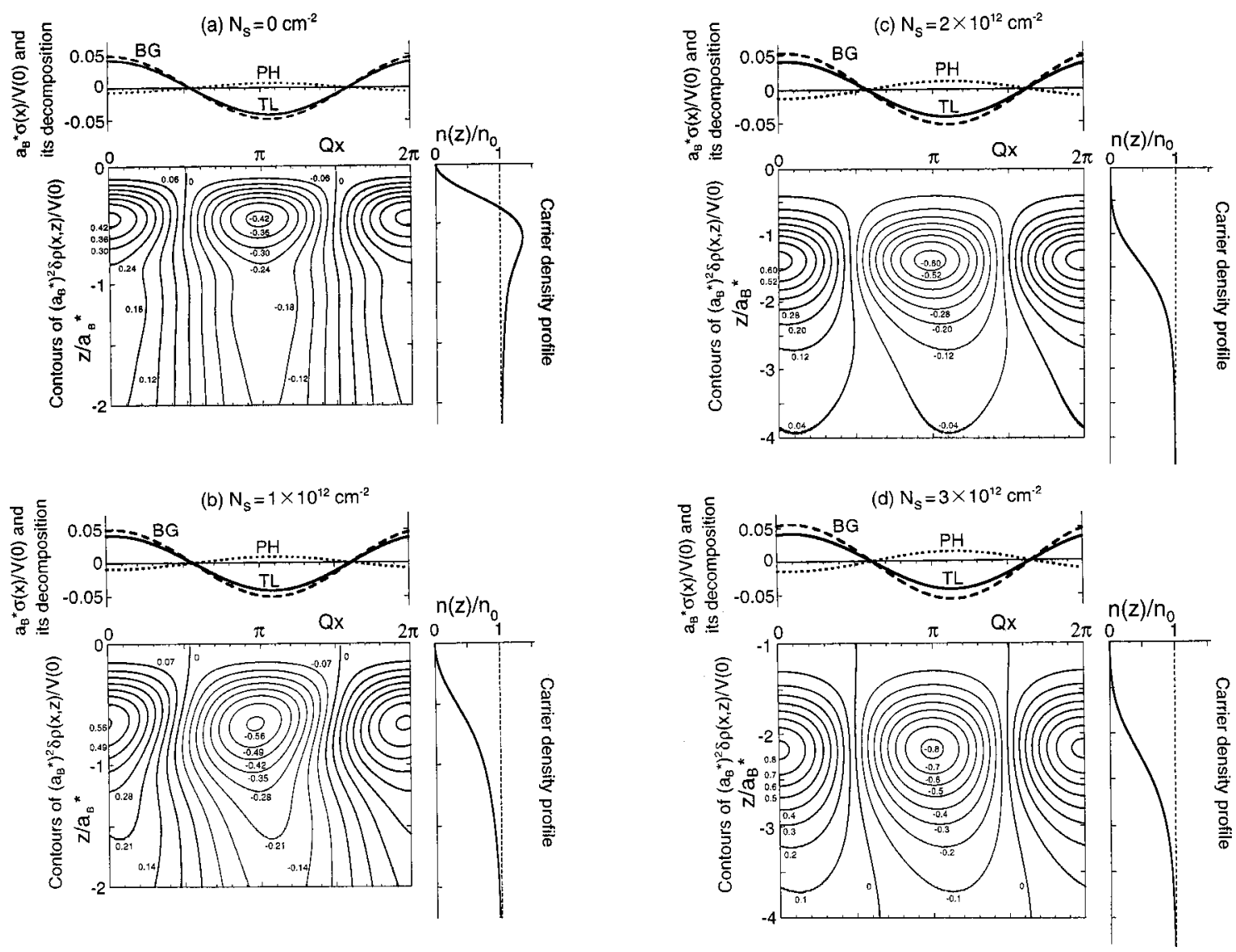

FIG. 4. Evolution of the induced charge-density distribution of the mode $A$ at $Q=3 \times 10^{5} \mathrm{~cm}^{-1}$ and the carrier-density distribution in thermal equilibrium in the depletion-layer formation process. In each panel with the depleted carrier density $N_{s}$ specified, the upper part exhibits the distribution of the induced surface-charge density (TL) and its decomposition into the background component (BG) and the phonon component $(\mathrm{PH})$. The left-lower area displays the contour map of the induced charge-density distribution below the surface. The right portion presents the carrier-density distribution in thermal equilibrium. The $x$ axis in the direction of $\mathbf{Q}$ and the $z$ axis normal to the surface are scaled in units of $Q^{-1}$ and $a_{B}^{*}$, respectively.

non component $\sigma_{\mathrm{PH}}$ grows in contribution, and its amplitude comes up to about one fourth of that of $\sigma_{\mathrm{BG}}$. The antiphase relation and the amplitude ratio of $\sigma_{\mathrm{BG}}$ and $\sigma_{\mathrm{PH}}$ are evident in the decomposition of $\sigma$ in Fig. 4 as well. The above growth in the amplitude ratio of $\delta \rho_{\mathrm{PH}}$ to $\delta \rho_{\mathrm{EL}}$ and $\sigma_{\mathrm{PH}}$ to $\sigma_{\mathrm{BG}}$ implies that, as the mode energy descends toward the phonon energy regime with increasing $N_{s}$, the phonon component begins to make more contribution to $\delta \rho$ and $\sigma$. In other words, plasmon character becomes less predominant with an increase in $N_{s}$. Generally, in coupled plasmonphonon modes of III-V compound semiconductors, polar phonons make less contribution to the energy-loss intensity than carrier plasmons, because polarity is not so strong. Accordingly, the above decline in plasmon character operates to reduce the surface energy-loss intensity. This is similar to the variation in the higher-energy one of the coupled modes with a decrease in doping level. As the mode energy falls toward the phonon energy regime with a decrease in $n_{0}$, plasmon character becomes less influential through coupling with polar phonons, and the integrated energy-loss intensity drops more significantly than it does in pure plasmon modes. This significant intensity drop is found both in the bulk ${ }^{57}$ and at the surface. ${ }^{45}$
To analyze phenomena only inside the material $(z \leqslant 0)$, we can assume the external potential to be generated by those external charges that lie right on the surface $(z=0)$. The added potential $\phi_{S}(0)+U(0)$, where $\phi_{S}(0)$ is defined by $\phi_{S}(0)=\phi_{\mathrm{SBG}}(0)+\phi_{\mathrm{SPH}}(0)$, is produced by the component of $\sigma$ that survives after canceling out the above external charges. In view of the fact that the complex value $\left[\phi_{S}(0)\right.$ $+U(0)] / U(0)$ has almost the same phase as $\left[\phi_{\mathrm{EL}}(0)\right.$ $\left.+\phi_{\mathrm{PH}}(0)\right] / U(0)$, we can realize that the variation in the surviving component of $\sigma$ along $x$ is almost coherent with that in $\delta \rho$ along $x$.

Next, we examine the contour map of the induced chargedensity distribution, along with the carrier-density profile in thermal equilibrium. At $N_{s}=0 \mathrm{~cm}^{-2}$, the main structure in contours of $\delta \rho(x, z)$ is an alternating array of a high mountain and a deep valley in a surface region $|z| / a_{B}^{*} \lesssim 0.8$, where the thermal-equilibrium carrier density $n$ rises rapidly from zero and forms a peak near the surface. The center of the main structure is located in a high-density region $\left(n \sim n_{0}\right)$ near the peak in the carrier-density profile. Each high mountain or each deep valley in the main structure has a long tail extending down into the deeper region. This extending feature results from the fact that the surface-mode energy is 

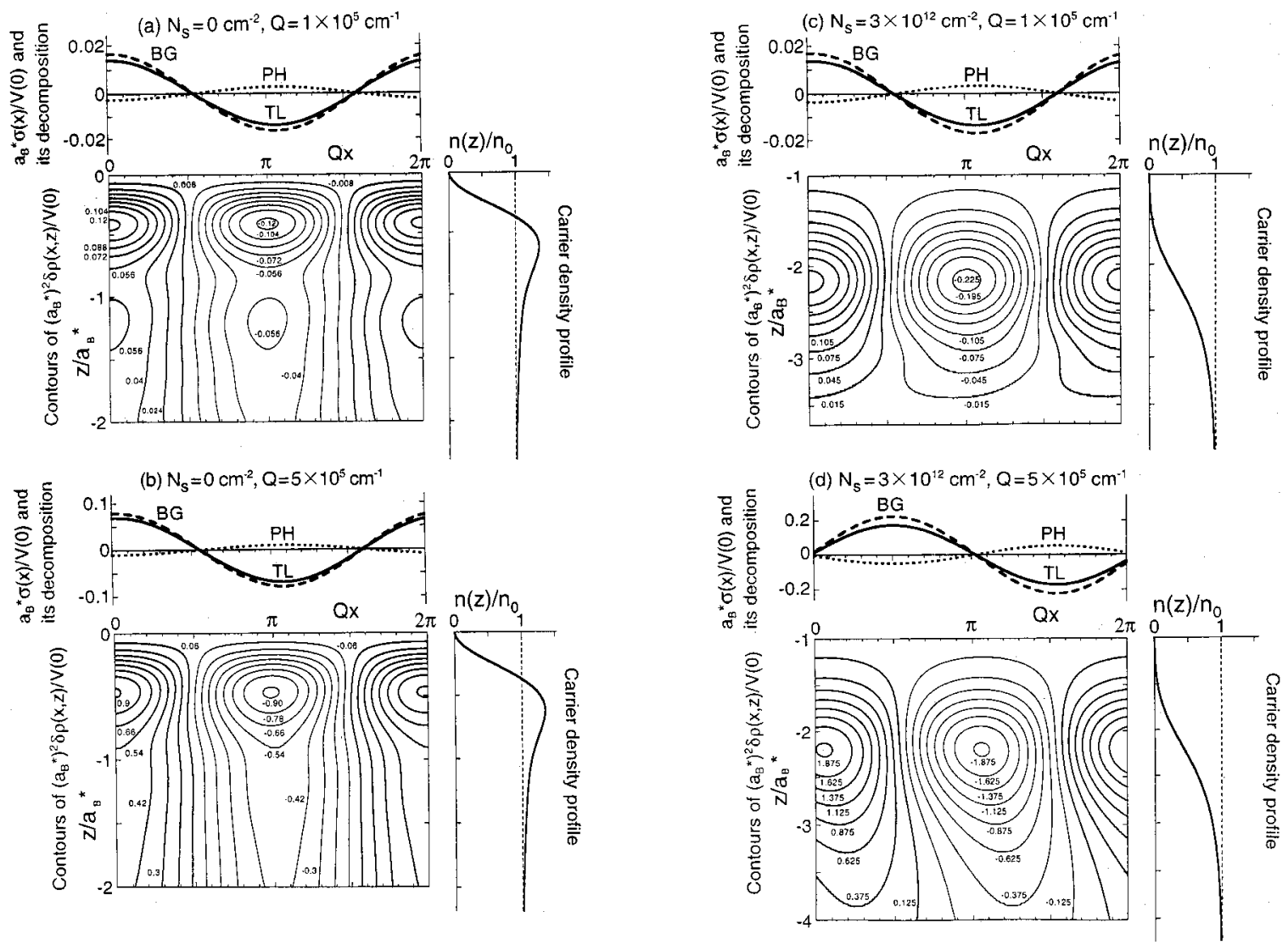

FIG. 5. Same as Fig. 4, but of the mode $A$ with (a) $N_{s}=0 \mathrm{~cm}^{-2}, Q=1 \times 10^{5} \mathrm{~cm}^{-1}$, (b) $N_{s}=0 \mathrm{~cm}^{-2}, Q=5 \times 10^{5} \mathrm{~cm}^{-1}$, (c) $N_{s}=3$ $\times 10^{12} \mathrm{~cm}^{-2}, Q=1 \times 10^{5} \mathrm{~cm}^{-1}$, and (d) $N_{s}=3 \times 10^{12} \mathrm{~cm}^{-2}, Q=5 \times 10^{5} \mathrm{~cm}^{-1}$.

slightly below or almost equal to the bottom of the projected bulk-mode band, because of high background polarization $\varepsilon_{\infty}(\gg 1)$. This fact can be understood by a simple evaluation using the local-response theory. In the absence of polar phonon, for example, the surface-plasmon frequency $\omega_{\mathrm{SPL}}$ at $Q$ $=0$ is expressed as $\omega_{\mathrm{SPL}}=\left[\varepsilon_{\infty} /\left(\varepsilon_{\infty}+1\right)\right]^{1 / 2} \omega_{\mathrm{BPL}}$, which is slightly below the bulk-plasmon frequency $\omega_{\mathrm{BPL}}$ at wave number $q=0$. As the surface-mode energy becomes further lower than the bulk-mode energies with an increase in $N_{s}$, the above extending feature dwindles away, and simultaneously, the main structure begins to extend in a broader $z$ range where the carrier density $n$ rises slowly toward the bulk value $n_{0}$ [follow Figs. 4(a) to 4(d)].

Here, we comment on a distorted feature in the main structure in Fig. 4(b). With an increase in $N_{s}$ from $0 \mathrm{~cm}^{-2}$ to $1 \times 10^{12} \mathrm{~cm}^{-2}$, the carrier density $n$ drops dramatically near the surface, and it starts to rise from zero slowly and monotonically without forming a peak. However, the surface potential barrier at $z=0$ still exerts a significant influence on carriers, as is evident from the fact that $n$ rises from zero just at the surface $z=0$ [see the right part of Fig. 4(b)]. The center of the main structure occurs still near the surface but in a lower-density region $\left(n \sim n_{0} / 2\right)$ where $n$ is increasing toward $n_{0}$. In this situation, a distorted feature appears in the main structure at $N_{s}=1 \times 10^{12} \mathrm{~cm}^{-2}$. This feature suggests that the center of the main structure tends to fall behind in propagating along the surface. As the main structure becomes separated from the surface with a further increase in
$N_{s}$, this distorted feature disappears, and the main structure evolves into those round patterns at a distance from the surface that lie in a wider $z$ range, where $n$ rises slowly and monotonically.

Here, we mention the relation between the induced charge-density distribution and the energy-loss intensity. The carrier component $\delta \rho_{\text {EL }}$ plays the leading role in the mode $A$ with strong plasmon character. As seen from Eqs. (19), (29), and (30), the imaginary part of $-\delta \rho_{\mathrm{EL}}(z) / U(0)$ is associated with the energy-loss intensity $F$. Because of the weight factor $e^{Q z}$ in Eq. (30), the induced charges $\delta \rho_{\mathrm{EL}}$ nearer to the surface make more contribution to $F$. At $N_{s}=0 \mathrm{~cm}^{-2}$, the main structure with concentrated induced charges just near the surface gives rise to stronger intensity of $F$. With the formation of the depletion layer, the induced charges become separated from the surface, which operates to reduce $F$. In addition, the above-stated decline in plasmon character acts to suppress the value of $\operatorname{Im}\left[-\delta \rho_{\mathrm{EL}}(z) / U(0)\right]$.

So far, we have analyzed the mode at $Q=3 \times 10^{5} \mathrm{~cm}^{-1}$ with change in $N_{s}$. Next, we turn our attention to the mode $A$ at other $Q$ values. Figure 5 exhibits the spatial structure of the mode $A$ with (a) $N_{s}=0 \mathrm{~cm}^{-2}, Q=1 \times 10^{5} \mathrm{~cm}^{-1}$, (b) $N_{s}$ $=0 \mathrm{~cm}^{-2}, Q=5 \times 10^{5} \mathrm{~cm}^{-1}$, (c) $N_{s}=3 \times 10^{12} \mathrm{~cm}^{-2}, Q=1$ $\times 10^{5} \mathrm{~cm}^{-1}$, and (d) $N_{s}=3 \times 10^{12} \mathrm{~cm}^{-2}, Q=5 \times 10^{5} \mathrm{~cm}^{-1}$. This figure is organized in the same fashion as Fig. 4. Irrespective of the $Q$ value, the contour lines for $N_{s}=0 \mathrm{~cm}^{-2}$ are constituted of the main structure with concentrated induced charges in a surface region and the tails extending down into 
the depth. As seen from Fig. 5(a), at $Q=1 \times 10^{5} \mathrm{~cm}^{-1}$, the extending tails present a perceptible oscillation that can be ascribed to the interference of electronic waves impinging on and reflected from the surface. However, this oscillation can hardly be perceived at a larger $Q$ value, $Q=5 \times 10^{5} \mathrm{~cm}^{-1}$ [see Fig. 5(b)]. In the light of considerable degeneracy of our carrier system, the period of oscillation at a smaller $Q$ value could be reasonably well scaled by $k_{F}^{-1}=2.96 \mathrm{~nm}\left(k_{F}^{-1} / a_{B}^{*}\right.$ $=0.306$ ). This oscillation can also be found in another dynamical-response calculation for $n_{0}=3 \times 10^{17} \mathrm{~cm}^{-3}$ and $T=300 \mathrm{~K}$, which assumes a flat band even near the surface and the Maxwell-Boltzmann distribution for carriers. ${ }^{58}$ In our previous calculation for $n$-type $\operatorname{InSb}$ at $T=0 \mathrm{~K}$, assuming a flat band at the surface, the extending contour lines show an outstanding oscillation due to the above-mentioned interference. ${ }^{44,45}$ However, the present result for $T=300 \mathrm{~K}$ exhibits only modest oscillations in the extending tails even at a smaller $Q$ value, because the temperature effect operates to smear out these oscillations. This is consistent with the temperature dependence of the carrier-density distribution $n(z)$ near the surface. ${ }^{36}$ At $T=0 \mathrm{~K}$, there is a small oscillation superimposed on the density curve approaching the bulk density. At $T=300 \mathrm{~K}$, however, thermal excitations up to higher electron states operate to wash out this density oscillation.

At $N_{s}=3 \times 10^{12} \mathrm{~cm}^{-2}$, each high mountain or each deep valley in the main structure has no tails extending into the deeper region. At $Q=1 \times 10^{5} \mathrm{~cm}^{-1}$, the main structure tends to be localized in a $z$ range, where $n$ rises slowly and monotonically, and displays signs of the above-mentioned oscillation only in contour lines of the lowest absolute value [see Fig. 5(c)]. With an increase in $Q$, however, the lower part of the main structure begins to extend downward, and the signs of the oscillation disappear. At $Q=5 \times 10^{5} \mathrm{~cm}^{-1}$, distorted features of contour lines imply that the lower extending part tends to go forward in propagating along the surface [see Fig. 5(d)]. This extending feature could be attributed to the Landau damping, namely, the damping effect of singleparticle excitations between semi-infinite electron states on plasmons, because the Landau damping is found to operate effectively in this mode, as shown in Fig. 1(c). In addition, the Landau damping is also responsible for the fact that the variation in $\sigma(x)$ in Fig. 5(d) is obviously different in phase from that in any other panel in Figs. 4 and 5. In the case of Fig. 5(d), because of the Landau damping, the positive imaginary part of $\varepsilon_{S}(\mathbf{Q}, \omega)$ becomes significantly larger than $\left|\operatorname{Re} \varepsilon_{S}(\mathbf{Q}, \omega)\right|$, and the argument of $\varepsilon_{S}(\mathbf{Q}, \omega)$ becomes close to $\pi / 2$ radian. Here, the symbol Re signifies the real part. This is in sharp contrast to any other case where $\operatorname{Im} \varepsilon_{S}(\mathbf{Q}, \omega)$ is small compared with $\left|\operatorname{Re} \varepsilon_{S}(\mathbf{Q}, \omega)\right|(\approx 1)$, and $\varepsilon_{S}(\mathbf{Q}, \omega)$ has an argument close to $\pi$ radian. Equations (26) and (27) assert that the difference in argument of $\varepsilon_{S}(\mathbf{Q}, \omega)$ gives rise to that in phase of $\sigma(x)$.

In view of the above result, we analyze the variation of the higher-energy loss peak in the EEL spectrum in Fig. 2 of Ref. 35. For this analysis, first we need to locate a dispersion region probed in this EELS measurement in the specularreflection geometry. The EEL intensity at each energy $\hbar \omega$ can be obtained by integrating in $\mathbf{Q}$ the product of a kinematic factor $K(\mathbf{Q}, \omega)$ and the surface energy-loss function $F(\mathbf{Q}, \omega)$ (see, e.g., Eqs. (2.1) and (2.3) in Ref. 41). The kinematic factor $K(\mathbf{Q}, \omega)$ depends upon the scattering process of an incident electron, and consequently, upon the incident angle $\alpha$ and the incident energy $E_{0}$. At each $\hbar \omega$ value, we can evaluate a probed $Q$ region from an ellipselike area on the $\mathbf{Q}$ plane where $K$ assumes values larger than half its maximum. From this evaluation for $\alpha=45^{\circ}$ and $E_{0}=9 \mathrm{eV}$ in Ref. 35, we find that, at $\hbar \omega \approx 48 \mathrm{meV}$, the probed region spreads from $Q \approx 2.0 \times 10^{5} \mathrm{~cm}^{-1}$ to $Q \approx 5.6 \times 10^{5} \mathrm{~cm}^{-1}$. This probed region, which is rather broad, is included in the dispersion diagram of Fig. 2. With an increase in $N_{s}$, the higher-energy loss peak in the EEL spectrum shows a downward shift in energy and a conspicuous decline in intensity. The downward shift in the dispersion branch with an increase in $N_{s}$ corresponds to that in the loss peak in the spectrum. With formation of the depletion layer, the separation of the carrier system from the surface and the decline in plasmon character cooperate to reduce the surface energy-loss intensity. In addition, the Landau damping also starts to operate effectively to suppress it in a larger $Q$ region. These cooperative effects explain the remarkable decline in the loss-peak intensity in the spectrum.

\section{B. Lower-energy mode $B$}

This is the lower-energy one of the two coupled plasmonphonon modes. Figure 6 displays the $\omega$ dependence of $F$ of the lower-energy mode $B$ at $Q=2 \times 10^{5} \mathrm{~cm}^{-1}$ (a) and 4 $\times 10^{5} \mathrm{~cm}^{-1}$ (b). This figure is laid out in the same manner as Fig. 1. With an increase in $N_{s}$, the mode energy shows a downward shift at each $Q$ value, and the resonance peak broadens remarkably at a larger $Q$ value [see Fig. 6(b)]. This broadening originates from the Landau damping. The above behavior with change in $N_{s}$ is similar to that in the mode $A$. However, in the mode $B$, the downward shift is small, and the resonance intensity is weak, compared with those in the mode $A$. In addition, in the mode $B$, we can find no definite decrease or increase in its integrated energy-loss intensity with change in $N_{s}$. As mentioned below, this intensity retention can be attributed to a few competing effects. Figure 7 presents the evolution of the energy dispersion of the mode $B$ in the depletion-layer formation process. At our considerably high carrier concentration $\left(n_{0}=1.3 \times 10^{18} \mathrm{~cm}^{-3}\right)$, the dispersion curve of the mode $B$ lies only a few meV below the transverse optical-phonon energy $\hbar \omega_{\mathrm{TO}}=33.25 \mathrm{meV}$. With an increase in $N_{s}$, the dispersion curve makes a gradual descent, and an upward dispersion at $N_{s}=0 \mathrm{~cm}^{-2}$ turns into a warped dispersion. This variation in the dispersion is similar to that in the mode $A$, aside from the fact that the energy shift is small in the mode $B$ with a considerable phonon component. Figures 8 and 9 show the variation in $\phi_{\mathrm{EL}}(0) / U(0)$, $\phi_{\mathrm{PH}}(0) / U(0), \phi_{\mathrm{SBG}}(0) / U(0)$, and $\phi_{\mathrm{SPH}}(0) / U(0)$, and that in the induced charge-density distribution of the mode $B$ at $Q=2 \times 10^{5} \mathrm{~cm}^{-1}$, respectively. These figures conform to the same formats as in Figs. 3 and 4, respectively. 

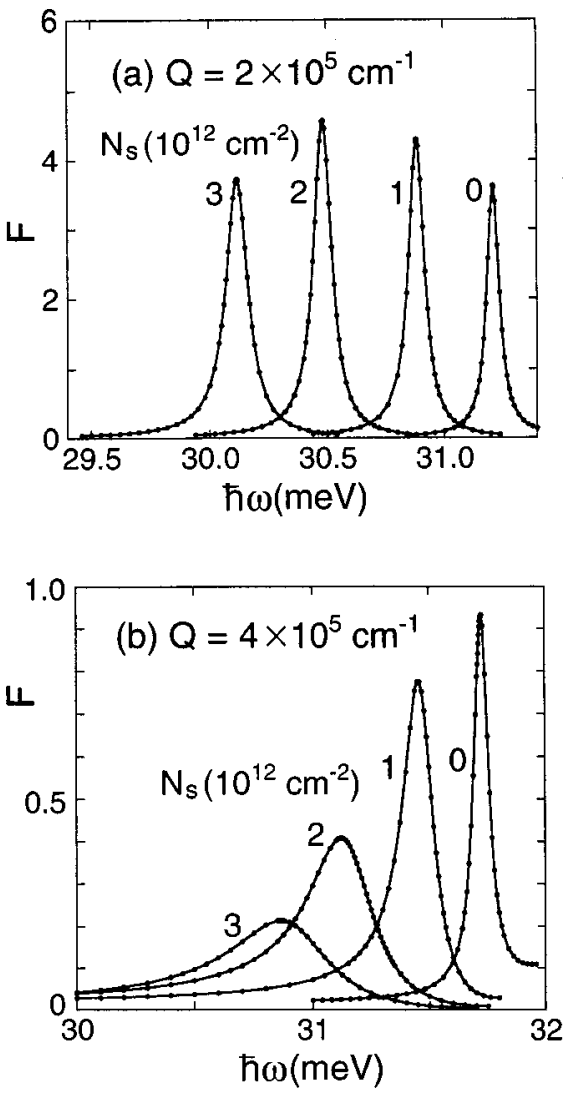

FIG. 6. $\omega$ dependence of the surface energy-loss function $F$ of the lower-energy mode $B$ at $Q=2 \times 10^{5} \mathrm{~cm}^{-1}$ (a) and 4 $\times 10^{5} \mathrm{~cm}^{-1}$ (b). The numeral $0,1,2$, or 3 denotes the depleted carrier density $N_{s}$ in units of $10^{12} \mathrm{~cm}^{-2}$.

The coupling character in the mode $B$ stands in sharp contrast to that in the mode $A$. As seen from Fig. 8, irrespective of $N_{s}$, the variation in $\delta \rho_{\mathrm{EL}}(x, z)$ along $x$ is in an antiphase relation with that in $\delta \rho_{\mathrm{PH}}(x, z)$ along $x$, and the former has a larger amplitude than the latter. The carrier component $\delta \rho_{\mathrm{EL}}$ operates to enhance $F$, while the phonon component $\delta \rho_{\mathrm{PH}}$ acts to reduce it. Because of our considerably high carrier concentration, the mode energy is close to the phonon energy regime, and the contribution of $\delta \rho_{\mathrm{PH}}$ be-

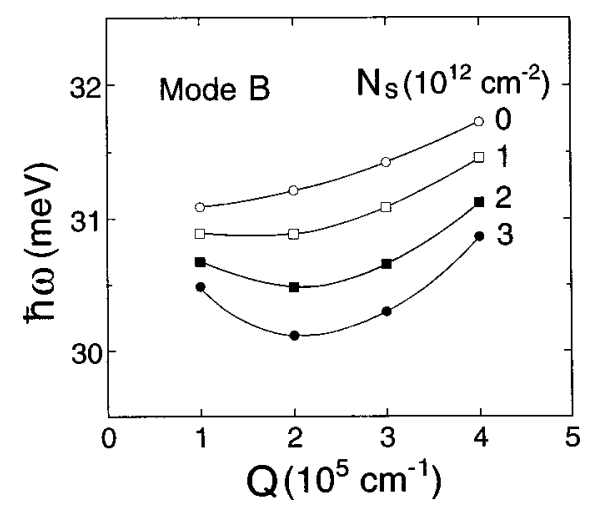

FIG. 7. Variation in the energy dispersion of the mode $B$ in the depletion-layer formation process. Organized in the same manner as Fig. 2. comes comparable to that of $\delta \rho_{\mathrm{EL}}$. Accordingly, there occurs a significant cancellation between $\delta \rho_{\mathrm{EL}}$ and $\delta \rho_{\mathrm{PH}}$, which leads to the weak energy-loss intensity of the mode $B$. However, we should note that $\delta \rho_{\mathrm{EL}}$ always makes more contribution to $\delta \rho$ than $\delta \rho_{\mathrm{PH}}$. As shown in Fig. 8, the variation in $\sigma_{\mathrm{PH}}(x)$ is in a coherent-phase relation with that in $\sigma_{\mathrm{BG}}(x)$, and both of these components operate to intensify $F$. The amplitude of $\sigma_{\mathrm{PH}}$ is comparable to that of $\sigma_{\mathrm{BG}}$ in the mode $B$, though the former is much smaller than the latter in the mode $A$ with strong plasmon character. With an increase in $N_{s}$, the amplitude ratio of $\delta \rho_{\mathrm{PH}}$ to $\delta \rho_{\mathrm{EL}}$ and that of $\sigma_{\mathrm{PH}}$ to $\sigma_{\mathrm{BG}}$ decrease gradually [follow Figs. 8(a) to 8(d)]. This implies that, as the mode energy falls further below the phonon energy regime with increasing $N_{s}$, phonon character becomes weaker, namely, the mode $B$ begins to acquire more plasmon character. This increase in plasmon character operates to enhance $F$, whereas the presence of the depletion layer separates the induced charges $\delta \rho$ from the surface, which acts to reduce $F$. In addition, the Landau damping also comes into play to suppress $F$ in a larger $Q$ region. These competing effects are considered to be responsible for the fact that the integrated energy-loss intensity of the mode $B$ shows no definite decrease or increase with change in $N_{s}$ (see Fig. 6). The above effect of the increasing plasmon character can also be recognized from the doping-level dependence of the lower-energy one of the coupled plasmonphonon modes. We are now concerned with a higherconcentration regime involving significant cancellation between $\delta \rho_{\mathrm{EL}}$ and $\delta \rho_{\mathrm{PH}}$. As the mode energy makes a gradual descent further below $\hbar \omega_{\text {TO }}$ with a decrease in $n_{0}$, plasmon character begins to grow in this mode with a weakening of the above cancellation, which gives rise to an outstanding enhancement of the integrated energy-loss intensity. This enhancement is found both in the bulk ${ }^{57}$ and at the surface. ${ }^{45}$

In the same manner as in the mode $A$, we can assume that the external charges lie right on the surface $(z=0)$, and consider the component of $\sigma$ that survives after canceling out the external charges. From an analysis of the phase relation in Fig. 8, we find that the variation in the surviving component of $\sigma$ along $x$ is nearly coherent with that in $\delta \rho$ along $x$. This is the same phase relation as in the mode $A$.

The evolution of the induced charge-density distribution with an increase in $N_{s}$ in Fig. 9 is similar to that in the mode $A$ in Fig. 4. As seen from Fig. 9(a), at $N_{s}=0 \mathrm{~cm}^{-2}$, the main structure having concentrated induced charges lies in a surface region $|z| / a_{B}^{*} \lesssim 0.8$, where $n$ rises quickly from zero and builds up a peak near the surface. This main structure has long tails extending down into the deeper region. Only in the initial stage of $N_{s}=1 \times 10^{12} \mathrm{~cm}^{-2}$, there appears a distorted feature in the main structure that implies that the center of the main structure tends to fall behind in propagating along the surface. As the mode energy descends gradually with an increase in $N_{s}$, the above extending feature becomes less conspicuous, and simultaneously, the main structure starts to extend in a thicker layer where $n$ rises slowly and monotonically toward its bulk value $n_{0}$. The reason why the spatial structure of the mode $B$ is similar to that of the mode $A$ with strong plasmon character is that, in the mode $B$ as well, the 
(a) $\mathrm{N}_{\mathrm{S}}=0 \mathrm{~cm}^{-2}$

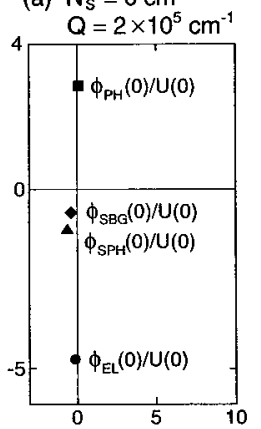

(b) $\mathrm{N}_{\mathrm{S}}=1 \times 10^{12} \mathrm{~cm}^{-2}$

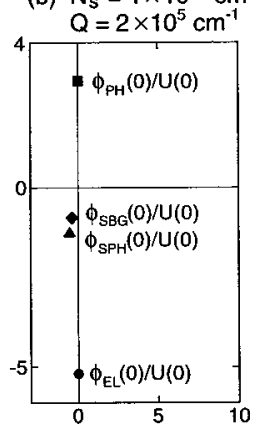

(c) $\mathrm{N}_{\mathrm{S}}=2 \times 10^{12} \mathrm{~cm}^{-2}$

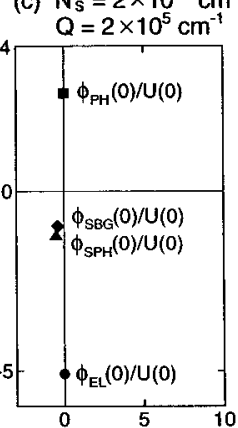

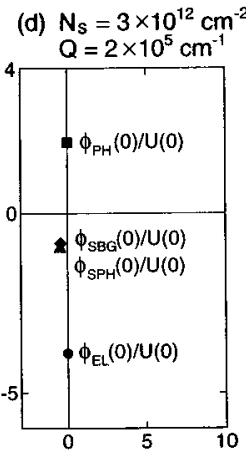

FIG. 8. Plot of $\phi_{\mathrm{EL}}(0) / U(0), \phi_{\mathrm{PH}}(0) / U(0)$, $\phi_{\mathrm{SBG}}(0) / U(0)$, and $\phi_{\mathrm{SPH}}(0) / U(0)$ of the mode $B$ at $Q=2 \times 10^{5} \mathrm{~cm}^{-1}$ for four $N_{s}$ values specified. Organized in the same fashion as Fig. 3. carrier component $\delta \rho_{\mathrm{EL}}$ makes more contribution to $\delta \rho$ than the phonon component $\delta \rho_{\mathrm{PH}}$, though the mode energy is close to the phonon energy regime.

In Fig. 9, we have examined the mode $B$ at $Q=2$ $\times 10^{5} \mathrm{~cm}^{-1}$. Figure 10 exhibits the spatial structure of the mode $B$ with other $Q$ values, namely, with (a) $N_{s}=0 \mathrm{~cm}^{-2}$, $Q=1 \times 10^{5} \mathrm{~cm}^{-1}$, (b) $N_{s}=0 \mathrm{~cm}^{-2}, Q=4 \times 10^{5} \mathrm{~cm}^{-1}$, (c) $N_{s}=3 \times 10^{12} \mathrm{~cm}^{-2}, \quad Q=1 \times 10^{5} \mathrm{~cm}^{-1}, \quad$ and (d) $N_{s}=3$ $\times 10^{12} \mathrm{~cm}^{-2}, Q=4 \times 10^{5} \mathrm{~cm}^{-1}$. In these cases as well, we can find the similarity in spatial structure between the modes $A$ and $B$. Only in a smaller $Q$ region, we can observe a contour oscillation that stems from the electronic interference [see Figs. 10(a) and 10(c)]. Because of temperature effects, the contour oscillation for $N_{s}=0 \mathrm{~cm}^{-2}$ and $T=300 \mathrm{~K}$ in Fig. 10(a) is not so conspicuous as that obtained in our previous calculation for $n$-type $\mathrm{InSb}$ at $T=0 \mathrm{~K}$ assuming a flat band at the surface. ${ }^{45}$ In a mode with a larger $Q$ value in the presence of a substantial depletion layer [see Fig. 10(d)], the lower part of the main structure extends downward, and presents a distorted feature, which suggests that the lower extending part tends to go forward in propagating along the surface. This extending feature could be ascribed to the Landau damping. This damping also accounts for the fact that the variation in $\sigma(x)$ in Fig. 10(d) is evidently different in phase from that in any other panel of Figs. 9 and 10. We also notice an initial phase deviation in $\sigma(x)$ in Fig. 10(b).

Based upon the above result of the mode $B$, we can understand the variation in the lower-energy loss peak in the EEL spectrum of Ref. 35. At $\hbar \omega \approx 31 \mathrm{meV}$, the probed $Q$ region extends from $Q \approx 1.3 \times 10^{5} \mathrm{~cm}^{-1}$ to $Q \approx 3.6$
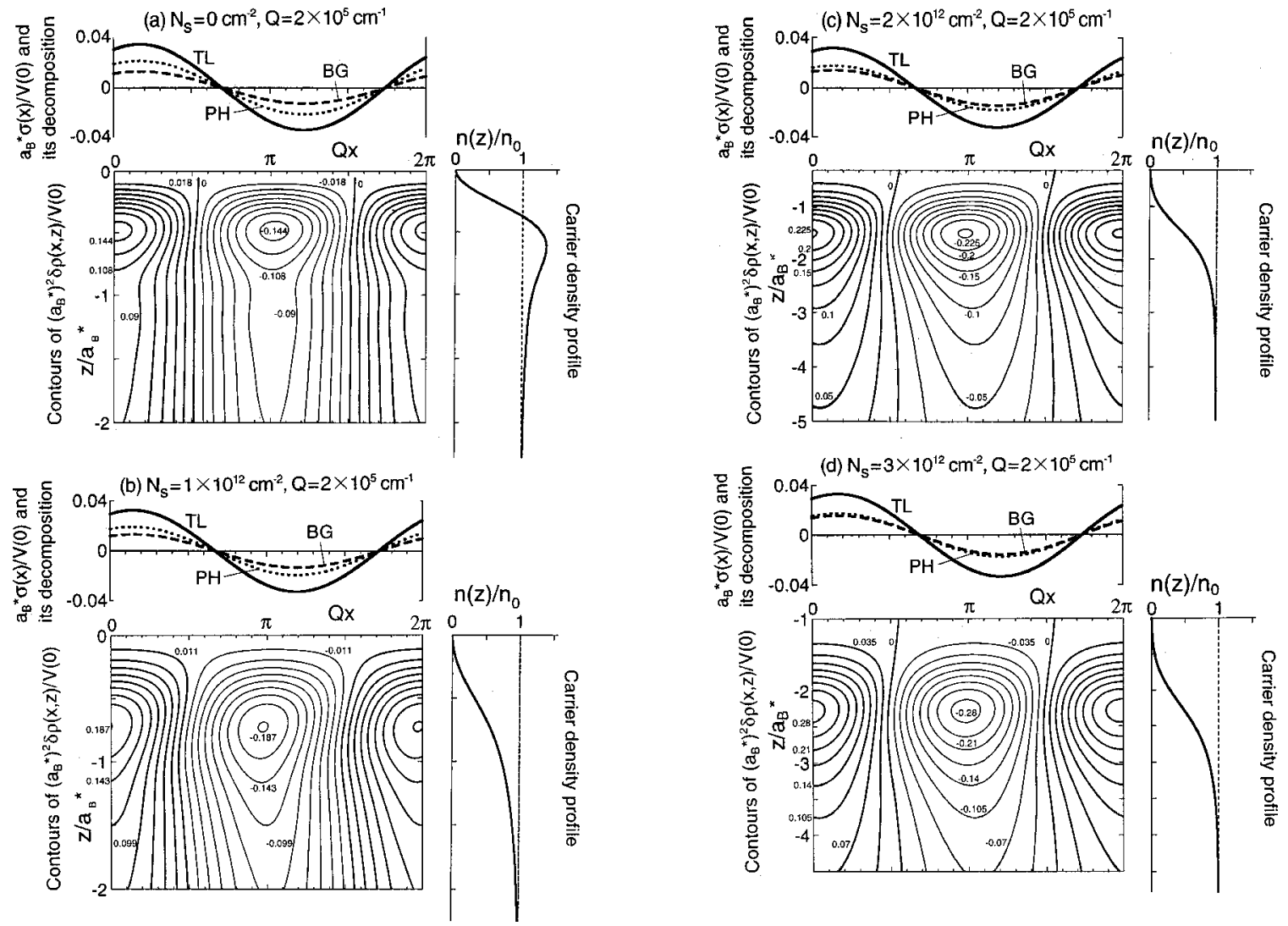

FIG. 9. Evolution of the induced charge-density distribution of the mode $B$ at $Q=2 \times 10^{5} \mathrm{~cm}^{-1}$ and the carrier-density distribution in thermal equilibrium in the depletion-layer formation process. Organized in the same way as Fig. 4. 

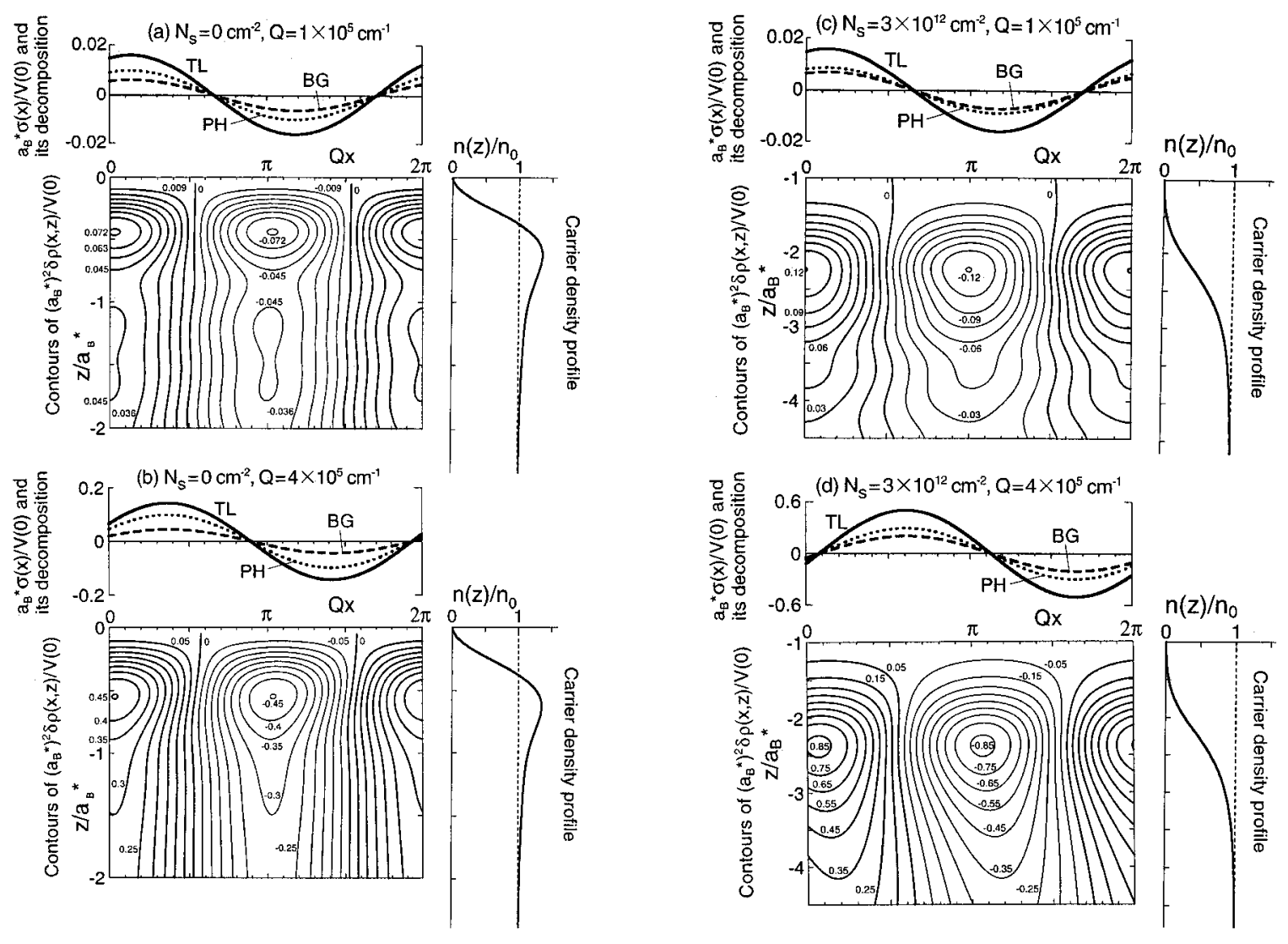

FIG. 10. Same as Fig. 9, but of the mode $B$ with (a) $N_{s}=0 \mathrm{~cm}^{-2}, Q=1 \times 10^{5} \mathrm{~cm}^{-1}$, (b) $N_{s}=0 \mathrm{~cm}^{-2}, Q=4 \times 10^{5} \mathrm{~cm}^{-1}$, (c) $N_{s}=3$ $\times 10^{12} \mathrm{~cm}^{-2}, Q=1 \times 10^{5} \mathrm{~cm}^{-1}$, and (d) $N_{s}=3 \times 10^{12} \mathrm{~cm}^{-2}, Q=4 \times 10^{5} \mathrm{~cm}^{-1}$.

$\times 10^{5} \mathrm{~cm}^{-1}$. With an increase in $N_{s}$, this loss peak in the spectrum exhibits a small downward shift in energy without any definite decline or growth in intensity. The small downward shift in the loss peak results from that in the dispersion curve in Fig. 7. The intensity retention with an increase in $N_{s}$ can be attributed to the above-mentioned competing effects in contribution to the energy-loss intensity. As seen from Fig. 6, the energy-loss intensity of the mode $B$ declines remarkably with an increase in $Q$ for each $N_{s}$ value. This is the same feature as in the mode $A$. The above probed $Q$ region lower than that for the mode $A$ is favorable to collecting the energy-loss intensity of the mode $B$ weaker than that of the mode $A$.

\section{Intermediate-energy mode $C$}

The intermediate-energy mode $C$ is the surface opticalphonon mode that undergoes the screening effect due to presence of carriers. Figure 11 shows the $\omega$ dependence of $F$ of the mode $C$ at $Q=1 \times 10^{5} \mathrm{~cm}^{-1}$ (a), $3 \times 10^{5} \mathrm{~cm}^{-1}$ (b), and $5 \times 10^{5} \mathrm{~cm}^{-1}$ (c). The ordinate is scaled differently in these panels. The resonance peak of the mode $C$ grows remarkably, as $N_{s}$ increases at each $Q$ value, or as $Q$ becomes larger with $N_{s}$ fixed. At each $Q$ value, the resonance peak presents a sight or small downward shift with an increase in $N_{s}$. By locating the peak position of $F$ at various $Q$ values, we can obtain the energy dispersion displayed in Fig. 12. This figure is organized in the same fashion as Figs. 2 and 7. In Fig. 12, the symbols $\hbar \omega_{\mathrm{LO}}$ and $\hbar \omega_{\mathrm{SPH}}$ denote, respectively, the bulk longitudinal-optical-phonon energy and the surface opticalphonon energy of long wavelength, both in the absence of carriers. The mode energy descends toward $\hbar \omega_{\mathrm{SPH}}$, as $N_{s}$ increases at each $Q$ value, or as $Q$ becomes larger at each $N_{s}$ value. The energy descent with $N_{s}$ is conspicuous at a larger $Q$ value, and that with $Q$ is outstanding at a larger $N_{s}$ value. The mode energy is close to $\hbar \omega_{\text {LO }}$ in a small $Q$ region. As described below, the striking change in the energy-loss intensity and the small, but meaningful shift in the mode energy reflect the variation in the screening effect. Figure 13 displays the plot of $\phi_{S}(0) / U(0), \quad \phi_{\mathrm{PH}}(0) / U(0)$, $\phi_{\mathrm{EL}}(0) / U(0)$, and $V(0) / U(0)$ of the mode $C$ at $Q=3$ $\times 10^{5} \mathrm{~cm}^{-1}$ as a triangle, a square, a circle, and a diamond, respectively. The imaginary part of $-\phi_{S}(0) / U(0)\left[\phi_{S}(0)\right.$ $\left.=\phi_{\mathrm{SPH}}(0)+\phi_{\mathrm{SBG}}(0)\right]$ indicates the contribution of $\sigma$ $\left(=\sigma_{\mathrm{PH}}+\sigma_{\mathrm{BG}}\right)$ to $F$, and that of $-V(0) / U(0)$ is equal to the value of $F$. The four panels (a)-(d) with $N_{s}$ specified correspond to the four points at $Q=3 \times 10^{5} \mathrm{~cm}^{-1}$ in Fig. 12 .

Here, we mention how the surface optical-phonon mode suffers the screening effect due to the existence of carriers. In the absence of carriers, the surface phonon mode has its induced charges only at the surface $(z=0)$. These induced charges $\sigma$ arise from the termination of the phonon and background polarization at $z=0$. In the presence of carriers, however, induced charges $\delta \rho$ occur below the surface $z=0$ as well. Though the presence of carriers is essential to 

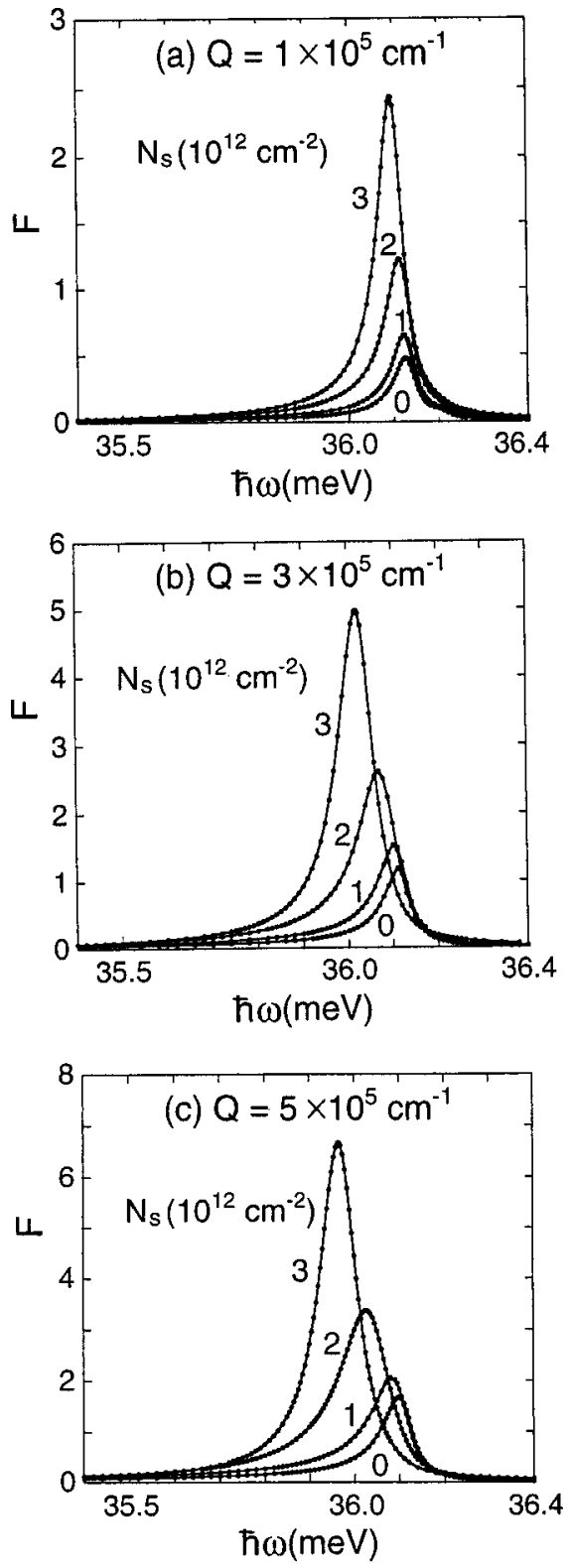

FIG. 11. $\omega$ dependence of the surface energy-loss function $F$ of the intermediate-energy mode $C$ at $Q=1 \times 10^{5} \mathrm{~cm}^{-1}$ (a), 3 $\times 10^{5} \mathrm{~cm}^{-1}(\mathrm{~b})$, and $5 \times 10^{5} \mathrm{~cm}^{-1}(\mathrm{c})$. Organized in the same manner as Figs. 1 and 6.

the occurrence of $\delta \rho$, it is not $\delta \rho_{\mathrm{EL}}$ but $\delta \rho_{\mathrm{PH}}$ that constitutes the major part of $\delta \rho$, as seen from the fact that $\left|\phi_{\mathrm{EL}}(0)\right|$ is quite small compared with $\left|\phi_{\mathrm{PH}}(0)\right|$ (see Fig. 13). The phonon plays the principal part in this mode. The induced charges $\delta \rho$ operate against $\sigma$ to reduce $F$, because $\operatorname{Im}\left[\left\{\phi_{\mathrm{PH}}(0)+\phi_{\mathrm{EL}}(0)\right\} / U(0)\right]$ is positive. This implies that the induced charges $\delta \rho$ exert the screening effect on the surface optical phonon governed by $\sigma$.

As shown in Fig. 13(a), at $N_{s}=0 \mathrm{~cm}^{-2}$, a negative imaginary part of $\phi_{S}(0) / U(0)$ and a positive one of $\left\{\phi_{\mathrm{PH}}(0)\right.$ $\left.+\phi_{\mathrm{EL}}(0)\right\} / U(0)$ tend to cancel out each other significantly. This indicates a strong screening effect, which leads to a very small value of $F$. With increasing $N_{s}$, however, the above cancellation becomes weaker, which results in a re-

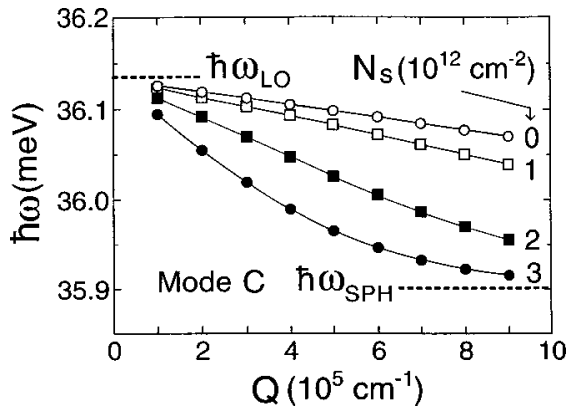

FIG. 12. Variation in the energy dispersion of the mode $C$ in the depletion-layer formation process. Organized in the same manner as Figs. 2 and 7. The symbols $\hbar \omega_{\mathrm{LO}}$ and $\hbar \omega_{\mathrm{SPH}}$ signify, respectively, the bulk longitudinal-optical-phonon energy and the surface opticalphonon energy of long wavelength, both in the absence of carriers.

markable enhancement of $F$. As the depletion layer becomes thicker, the screening charges $\delta \rho$ becomes further separated from $\sigma$, and the screening of $\delta \rho$ on $\sigma$ operates less effectively. This is responsible for the growth in the resonance peak in Fig. 11 and the downward shift in the mode energy in Fig. 12 with an increase in $N_{s}$.

The screening effect depends upon $Q$ remarkably, because the induced potential produced by the screening charges $\delta \rho$ attenuates exponentially with a decay distance $Q^{-1}$ in the carrier-deficient layer above $\delta \rho$. As the screening of $\delta \rho$ on $\sigma$ operates less effectively with an increase in $Q$, the mode energy descends toward $\hbar \omega_{\mathrm{SPH}}$ and the energy-loss intensity becomes stronger. This variation in energy and intensity is more prominent for a thicker depletion layer.

As in the analysis of the mode $A$ or $B$, we can assume that the external charges exist right on the surface $(z=0)$, and examine the component of $\sigma$ that survives after canceling out the external charges. The phase relation in Fig. 13 indicates that the variation in the surviving component of $\sigma$ along $x$ is almost in antiphase relation with that in $\delta \rho$ along $x$, in accordance with the result in our previous calculation, assuming a flat band at the surface. ${ }^{45}$ This antiphase relation in sharp contrast to the coherent-phase relation in the mode $A$ or $B$ leads to a nearly vertical electric field in a layer intermediate between $\sigma$ and $\delta \rho .^{45}$

Next, we focus our attention on the induced chargedensity distribution of the mode $C$ at $Q=3 \times 10^{5} \mathrm{~cm}^{-1}$. Figure 14 conforms to the same format as in Figs. 4 and 9. The panels (a) and (b) are for $N_{s}=0 \mathrm{~cm}^{-2}$ and $3 \times 10^{12} \mathrm{~cm}^{-2}$, respectively. Regardless of $N_{s}$, the variation in $\sigma_{\mathrm{PH}}(x)$ and that in $\sigma_{\mathrm{BG}}(x)$ tend to cancel out each other strongly, and the amplitude of $\sigma_{\mathrm{PH}}(x)$ is somewhat larger than that of $\sigma_{\mathrm{BG}}(x)$. In fact, this antiphase relation follows from Eq. (28) with the aid of Eq. (3), when the mode frequency $\omega$ is higher than $\omega_{\mathrm{TO}}$, and the relaxation-rate constant $\gamma$ is not so large. Irrespective of $N_{s}$, contour lines of $\delta \rho(x, z)$ present outstanding tails streaming in the opposite direction to $\mathbf{Q}$. These streaming tails indicate that the surface optical phonon propagates along the surface dragging the screening charges $\delta \rho$. Figure 15 clarifies the evolution of $\delta \rho$ of the mode $C$ at $Q=3$ $\times 10^{5} \mathrm{~cm}^{-1}$ with a change in $N_{s}$. The left and middle panels exhibit the $z$ dependence of the absolute value and the argu- 
(a) $\mathrm{N}_{\mathrm{s}}=0 \mathrm{~cm}^{-2}$

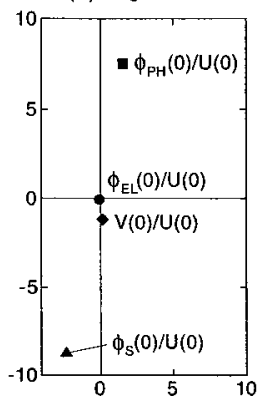

(b) $\mathrm{N}_{\mathrm{S}}=1 \times 10^{12} \mathrm{~cm}^{-2}$

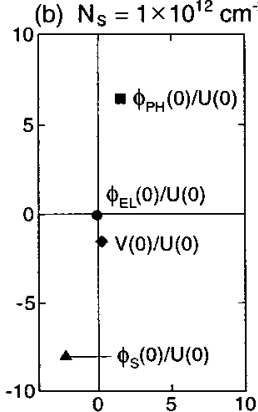

(c) $\mathrm{N}_{\mathrm{s}}=2 \times 10^{12} \mathrm{~cm}^{-2}$

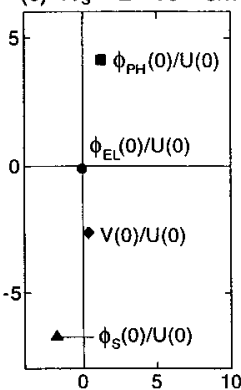

(d) $\mathrm{N}_{\mathrm{s}}=3 \times 10^{12} \mathrm{~cm}^{-2}$

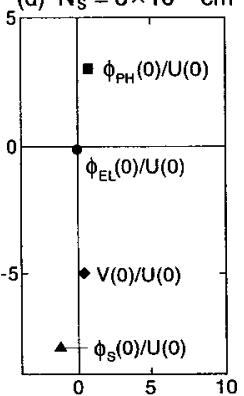

FIG. 13. Plot of $\phi_{S}(0) / U(0), \phi_{\mathrm{PH}}(0) / U(0)$, $\phi_{\mathrm{EL}}(0) / U(0)$, and $V(0) / U(0)$ of the mode $C$ at $Q=3 \times 10^{5} \mathrm{~cm}^{-1}$ for four $N_{s}$ values specified. The potential $\phi_{S}(0)$ is defined by $\phi_{S}(0)$ $=\phi_{\mathrm{SPH}}(0)+\phi_{\mathrm{SBG}}(0)$. ment $(\arg )$ of $\left(a_{B}^{*}\right)^{2} \delta \rho(\mathbf{Q}, z, \omega) / V(0)$, respectively. These panels elucidate the amplitude and the phase of the spatial variation in $\delta \rho$ at each depth $z$. In the left panel, the value of $\left(a_{B}^{*}\right)^{2}|\delta \rho(\mathbf{Q}, z, \omega) / V(0)|$ is doubled in two broken curves, as marked by the sign ' $\times 2$ '. The spatial variation in $\delta \rho$ in these panels can be compared with the carrier-density distribution $n(z)$ in thermal equilibrium in the right panel. In each panel, the numeral $0,1,2$, or 3 on each curve represents the $N_{s}$ value in units of $10^{12} \mathrm{~cm}^{-2}$. At a larger $N_{s}$ value, the screening charges $\delta \rho$ are distributed in a thicker layer, as is consistent with the fact that $n$ rises from zero more slowly near the surface, and with the fact that the screening of $\delta \rho$ on $\sigma$ operates less effectively. At $N_{s}=0 \mathrm{~cm}^{-2}$, the peak of $\left(a_{B}^{*}\right)^{2}|\delta \rho(\mathbf{Q}, z, \omega) / V(0)|$ is located at a depth of higher $n$,
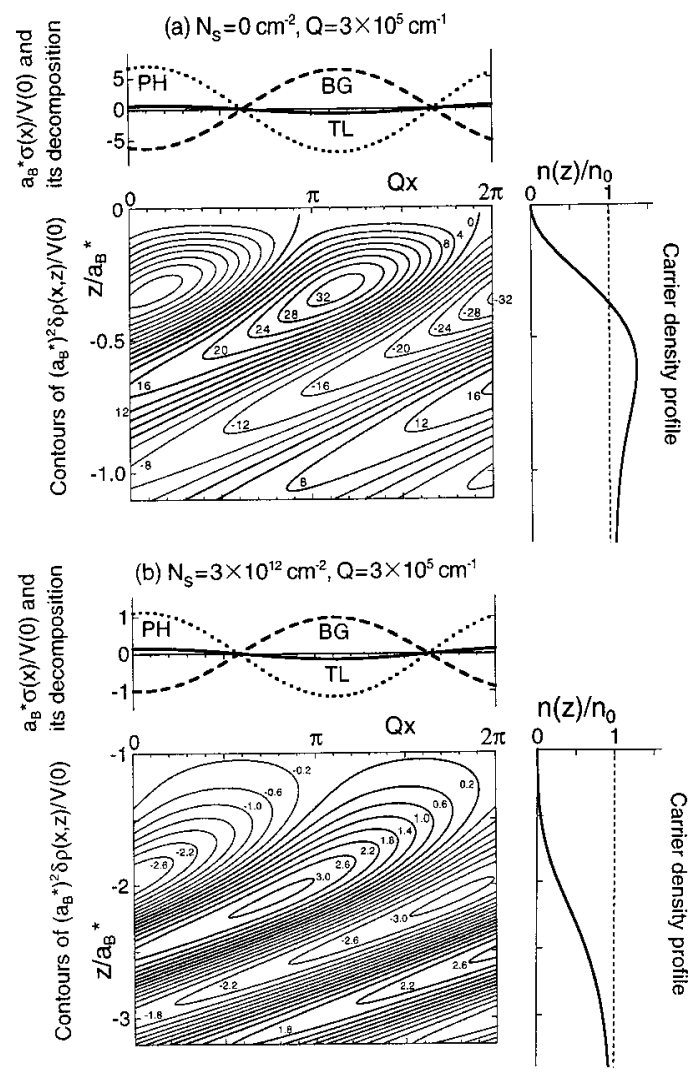

FIG. 14. Induced charge-density distribution of the mode $C$ at $Q=3 \times 10^{5} \mathrm{~cm}^{-1}$ and the carrier-density distribution in thermal equilibrium for $N_{s}=0 \mathrm{~cm}^{-2}$ (a) and $3 \times 10^{12} \mathrm{~cm}^{-2}$ (b). Organized in the same fashion as Figs. 4 and 9. while, at any other $N_{s}$ value, it is situated at a depth where $n$ is still on its initial rise and lower. When $N_{s}$ increases from 0 $\mathrm{cm}^{-2}$ to $1 \times 10^{12} \mathrm{~cm}^{-2}$, there occurs no substantial shift in the above peak, because, at the stage of $N_{s}=1 \times 10^{12} \mathrm{~cm}^{-2}$, the surface potential barrier at $z=0$ still plays an important role in restricting carriers to the inside of the material. With a further increase in $N_{s}$, the carriers begin to be confined in the inside by the upward-bending effective potential, and the peak represents a significant shift to the depth. As seen from the middle panel, at each $N_{s}$ value, the argument of $\delta \rho(\mathbf{Q}, z, \omega) / V(0)$ shows a marked monotonical increase on the deeper side of the peak of $|\delta \rho(\mathbf{Q}, z, \omega) / V(0)|$. This phase change corresponds to the fact that the surface optical phonon propagates along the surface dragging the screening charges $\delta \rho$. The argument increase is somewhat slower at a larger $N_{s}$ value. This may imply that the dragging declines at a larger $N_{s}$ value where the screening effect becomes weaker.

So far, we have investigated the spatial structure of the mode $C$ at $Q=3 \times 10^{5} \mathrm{~cm}^{-1}$. Here, we turn our attention to the mode $C$ at other $Q$ values. Figure 16 displays the $z$ dependence of $\left(a_{B}^{*}\right)^{2}|\delta \rho(\mathbf{Q}, z, \omega) / V(0)|$ (left panel) and $\arg [\delta \rho(\mathbf{Q}, z, \omega) / V(0)]$ (middle panel) of the mode $C$ at $Q$ $=1 \times 10^{5} \mathrm{~cm}^{-1}$ and $9 \times 10^{5} \mathrm{~cm}^{-1}$ for each value of $N_{s}$ $=0 \mathrm{~cm}^{-2}$ and $3 \times 10^{12} \mathrm{~cm}^{-2}$. The numeral 1 or 9 on each curve indicates the $Q$ value in units of $10^{5} \mathrm{~cm}^{-1}$, and full curves and broken ones represent the results for $N_{s}$ $=0 \mathrm{~cm}^{-2}$ and $3 \times 10^{12} \mathrm{~cm}^{-2}$, respectively. In the left panel,

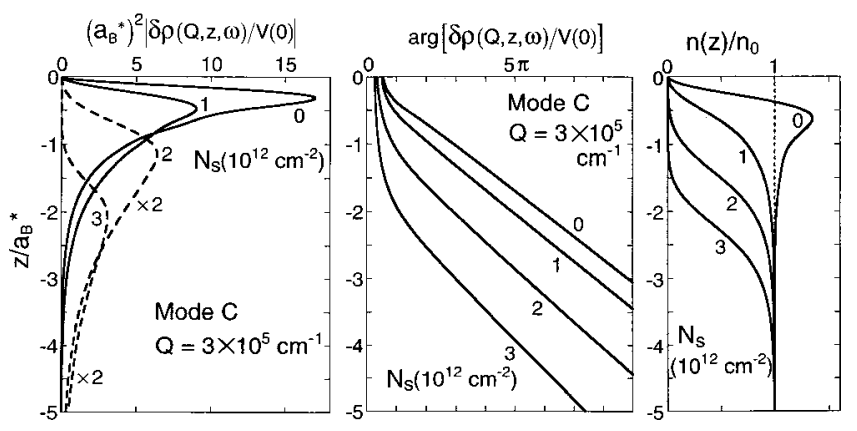

FIG. 15. $z$ dependence of $\left(a_{B}^{*}\right)^{2}|\delta \rho(\mathbf{Q}, z, \omega) / V(0)|$ (left panel) and $\arg [\delta \rho(\mathbf{Q}, z, \omega) / V(0)]$ (middle panel) of the mode $C$ at $Q=3$ $\times 10^{5} \mathrm{~cm}^{-1}$ in the depletion-layer formation process. The numeral $0,1,2$, or 3 on each curve signifies the depleted carrier density $N_{s}$ in units of $10^{12} \mathrm{~cm}^{-2}$. Compared with the carrier-density distribution in thermal equilibrium in the right panel. 

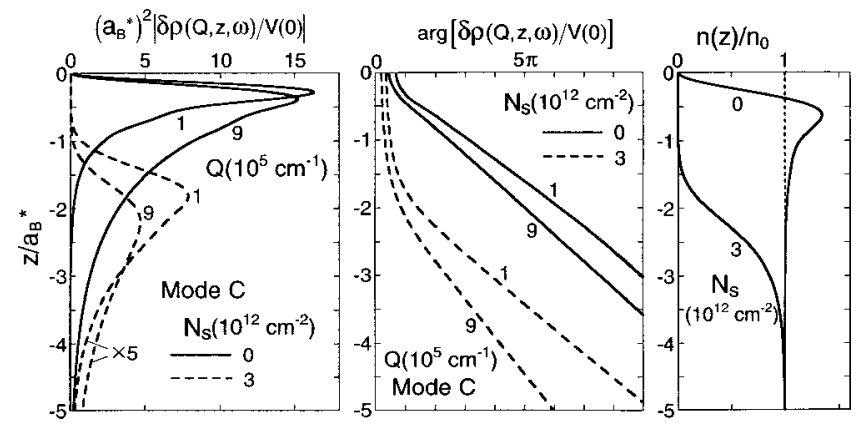

FIG. 16. Same as Fig. 15, but of the mode $C$ with other $Q$ values. In the left and middle panels, the numeral 1 or 9 on each curve designates the $Q$ value in units of $10^{5} \mathrm{~cm}^{-1}$, and full curves and broken ones are for $N_{s}=0 \mathrm{~cm}^{-2}$ and $3 \times 10^{12} \mathrm{~cm}^{-2}$, respectively.

the value of $\left(a_{B}^{*}\right)^{2}|\delta \rho(\mathbf{Q}, z, \omega) / V(0)|$ is multiplied by five in two broken curves. The four modes with various sets of $N_{s}$ and $Q$ can be located in the dispersion diagram of Fig. 12 . Figure 16 shows that the distribution of $\delta \rho$ for each $N_{s}$ value depends upon $Q$ significantly. This $Q$ dependence can be related to that of the screening effect. When the screening of $\delta \rho$ on $\sigma$ operates more effectively at a smaller $Q$ value, the greater part of the electric field produced by $\sigma$ and $\delta \rho$ is confined in a layer that intervenes between $\sigma$ and $\delta \rho$. In this case, the mode energy becomes closer to $\hbar \omega_{\mathrm{LO}}$, as exhibited in Fig. 12, and the screening charges $\delta \rho$ are more localized with the amplitude maximum nearer to the surface, as displayed in the left panel of Fig. 16. This localization is quite conspicuous at $Q=1 \times 10^{5} \mathrm{~cm}^{-1}$ and $N_{s}=0 \mathrm{~cm}^{-2}$. This localized feature suggests that the screening charges $\delta \rho$ are strongly attracted to the surface $(z=0)$ by the intense electric field virtually normal to the surface. With an increase in $Q$, the screening effect becomes less powerful, and a larger fraction of the electric field generated by $\sigma$ leaks out above the surface. In this process, the screening charges $\delta \rho$ extend into the deeper region with a gradual shift in the amplitude maximum to the depth, and the dragging character declines gradually, as indicated by a slower increase in the argument.

Finally, we analyze the variation in the middle loss peak in the EEL spectrum of Ref. 35. At $\hbar \omega \approx 36 \mathrm{meV}$, the probed $Q$ range spreads from $Q \approx 1.5 \times 10^{5} \mathrm{~cm}^{-1}$ to $Q \approx 4.2$ $\times 10^{5} \mathrm{~cm}^{-1}$. With an increase in $N_{s}$, the loss peak in the EEL spectrum grows strikingly without any substantial shift. This intensity growth originates from the fact that, with an increase in the depletion-layer thickness, the screening of $\delta \rho$ on $\sigma$ operates less effectively, which leads to stronger energy-loss intensity. No substantial shift observed corresponds to the fact that the dispersion branch shows too small a downward shift to be detected. In addition, the loss peak in the spectrum is observed to gain stronger intensity, when the incident energy $E_{0}$ is lowered in the presence of a substantial depletion layer formed (see Fig. 3 in Ref. 35). In HREELS with the specular-reflection geometry, lowering $E_{0}$ is equivalent to shifting the probed $Q$ region to the higher $Q$ side. ${ }^{39}$ The above intensity growth with a decrease in $E_{0}$ can be ascribed to the fact that, with an increase in $Q$, the screening effect of $\delta \rho$ on $\sigma$ becomes weaker, which results in the enhancement of the energy-loss intensity.

Predicated upon the calculated results, we have analyzed the variation in the EEL spectrum with an increase in $N_{s}$. Incidentally, it is far beyond our computing resources to calculate the EEL spectrum itself on the basis of our calculational scheme, because calculating even one mode takes a lot of CPU time, and we need to calculate a great number of modes in the broad probed region.

\section{SUMMARY}

We have investigated the evolution of elementary excitations at a doped polar semiconductor surface in a depletionlayer formation process. The elementary excitations analyzed are two coupled plasmon-phonon modes and the surface optical-phonon mode involving screening charges. Our calculations correspond to an HREELS experiment of an $n$-type GaAs(110) surface on exposure to hydrogen at room temperature where three loss peaks are clearly resolved. The induced charge density $\delta \rho$ below the surface is constituted of the carrier component $\delta \rho_{\mathrm{EL}}$ due to carrier density fluctuation and the phonon component $\delta \rho_{\mathrm{PH}}$ originating from longitudinal polar-phonon polarization. The induced surface-charge density $\sigma$ owing to the termination of the polarization at the surface is composed of the background component $\sigma_{\mathrm{BG}}$ and the phonon component $\sigma_{\mathrm{PH}}$. The $x$ axis is oriented to the direction of the surface-parallel wave vector $\mathbf{Q}$, and the $z$ axis is taken to be normal to the surface so that the material extends in the region $z<0$. The surface energy-loss function $F(\mathbf{Q}, \omega)$ describes the intensity of the energy loss involved in the dynamical response of the surface to the external potential of $\mathbf{Q}$ and $\omega$. The evolution of each excitation mode is summarized as follows:

\section{A. Higher-energy mode $A$}

This is the upper one of the two coupled plasmon-phonon modes at the surface.

(i) With an increase in the depleted carrier density $N_{s}$, the energy-dispersion branch shifts downward, and an upward dispersion at $N_{s}=0 \mathrm{~cm}^{-2}$ evolves into a warped dispersion that is downward in a smaller $Q$ region and that turns upward in a larger $Q$ region. This warped dispersion stems from competition between the plasmon nature that tends toward an upward dispersion and the effect of the depletion layer that leans toward a downward dispersion.

(ii) In spatial variation along $x, \delta \rho_{\mathrm{EL}}(x, z)$ is coherent with $\delta \rho_{\mathrm{PH}}(x, z)$, whereas $\sigma_{\mathrm{BG}}(x)$ is in an antiphase relation with $\sigma_{\mathrm{PH}}(x)$, and $\sigma_{\mathrm{BG}}(x)$ has a larger amplitude. This coupling character is independent of $N_{s}$. At our considerably high carrier concentration, the carrier component $\delta \rho_{\mathrm{EL}}$ makes more contribution to $\delta \rho$ than the phonon component $\delta \rho_{\mathrm{PH}}$.

(iii) As the dispersion branch descends toward the phonon energy regime with increase in $N_{s}$, the phonon components $\delta \rho_{\mathrm{PH}}$ and $\sigma_{\mathrm{PH}}$ increase their contribution to $\delta \rho$ and $\sigma$, respectively. This indicates that phonon character grows, and plasmon character becomes less influential. 
(iv) With an increase in $N_{s}$, the separation of the carrier system from the surface and the decline in plasmon character begin to cooperate to reduce the surface energy-loss intensity significantly. In addition, the Landau damping also starts to operate effectively to suppress it in a larger $Q$ region.

(v) The descent of the dispersion branch in (i) and the reduction in the loss intensity in (iv) elucidate the experimental result that the corresponding loss peak in the EEL spectrum shows a gradual downward shift in energy and a striking decline in intensity with increase in $N_{s}$.

(vi) At $N_{s}=0 \mathrm{~cm}^{-2}$, the induced charge-density distribution $\delta \rho(x, z)$ is composed of the main structure with concentrated induced charges and the tails extending into the deeper region. The main structure lies in a surface region where the thermal-equilibrium carrier density $n$ rises quickly from zero and forms a prominent peak in the density profile. The extending features can be attributed to the fact that the surfacemode energy is slightly below or almost equal to the bottom of the bulk-mode energies because of high background polarization. As the surface-mode energy becomes further lower than the bulk-mode energies with an increase in $N_{s}$, the extending feature dwindles away, and the main structure starts to extend in a broader $z$ range where $n$ rises slowly and monotonically toward its bulk value.

(vii) In a smaller $Q$ range, there appear modest or slight oscillations in contours of $\delta \rho(x, z)$ that can be ascribed to the interference of electronic waves impinging on and reflected from the surface.

(viii) In the presence of a substantial depletion layer, with an increase in $Q$, the lower part of the main structure in $\delta \rho(x, z)$ starts to extend downward and to show distorted features. This variation could be attributed to the Landau damping.

(ix) The Landau damping causes a phase shift in variation of $\sigma(x)$ in a larger $Q$ region and in the presence of a substantial depletion layer.

\section{B. Lower-energy mode $B$}

This is the lower one of the two coupled plasmon-phonon modes at the surface.

(i) The mode $B$ displays a similar variation in the energy dispersion to the mode $A$. With an increase in $N_{s}$, the dispersion branch descends, and an upward dispersion at $N_{s}$ $=0 \mathrm{~cm}^{-2}$ changes into a warped dispersion. At our considerably high carrier concentration, the dispersion is weak, and the downward shift is small, compared with those of the mode $A$ with strong plasmon character.

(ii) The coupling character of the mode $B$ is in sharp contrast to that of the mode $A$. In spatial variation along $x, \delta \rho_{\mathrm{EL}}(x, z)$ is in an antiphase relation with $\delta \rho_{\mathrm{PH}}(x, z)$, and $\delta \rho_{\mathrm{EL}}(x, z)$ has a larger amplitude, while $\sigma_{\mathrm{PH}}(x)$ is coherent with $\sigma_{\mathrm{BG}}(x)$. This coupling character remains unchanged with change in $N_{s}$. The predominance of $\delta \rho_{\mathrm{EL}}$ over $\delta \rho_{\mathrm{PH}}$ in amplitude underlies various similarities of the mode $B$ to the mode $A$ in energy dispersion and in spatial structure. At our considerably high carrier concentration, the above variation in $\delta \rho_{\mathrm{EL}}(x, z)$ and that in $\delta \rho_{\mathrm{PH}}(x, z)$ tend to cancel out each other significantly. (iii) As the dispersion branch descends further below the phonon energy regime with an increase in $N_{s}$, the phonon components $\delta \rho_{\mathrm{PH}}$ and $\sigma_{\mathrm{PH}}$ decrease their contribution to $\delta \rho$ and $\sigma$, respectively. This implies that phonon character declines, and plasmon character becomes more influential.

(iv) With an increase in $N_{s}$, the growth in plasmon character begins to operate to intensify $F$, whereas the separation of the carrier system from the surface starts to act to suppress it. In a larger $Q$ region, the Landau damping also begins to function to reduce it. These competing effects are responsible for the fact that the surface energy-loss intensity shows no definite increase or decrease with change in $N_{s}$.

(v) The small downward shift in the dispersion branch in (i) and the retention of the loss intensity in (iv) explain the experimental result that, with an increase in $N_{s}$, the corresponding loss peak in the EEL spectrum represents a small downward shift in energy and no definite increase or decrease in intensity.

(vi) With an increase in $N_{s}$, the mode $B$ displays a similar variation in $\delta \rho(x, z)$ to the mode $A$. At $N_{s}=0 \mathrm{~cm}^{-2}$, the induced charge-density distribution $\delta \rho(x, z)$ is constituted of the main structure with concentrated induced charges and the tails extending into the deeper region. As the mode energy drops gradually with an increase in $N_{s}$, the extending feature becomes less conspicuous, and the main structure begins to extend in a wider $z$ range.

(vii) In a smaller $Q$ region, there emerge those oscillations in contours of $\delta \rho(x, z)$ due to the electronic interference that are similar to those in the mode $A$.

(viii) When there exists a substantial depletion layer, with an increase in $Q$, the lower part of the main structure begins to extend downward and to exhibit distorted features. This variation, similar to that in the mode $A$, could be ascribed to the Landau damping.

(ix) As in the mode $A$, the Landau damping gives rise to a phase shift in variation of $\sigma(x)$ in a larger $Q$ region, especially in the presence of a substantial depletion layer.

\section{Intermediate-energy mode $C$}

This is the surface optical-phonon mode that suffers the screening effect due to the presence of carriers. The induced surface charges $\sigma$, which dominate the surface optical phonon, operate to intensity $F$, whereas the induced internal charges $\delta \rho$ due to the presence of carriers act to reduce it. This indicates that the induced charges $\delta \rho$ have the screening effect on the surface optical phonon governed by $\sigma$.

(i) The screening of $\delta \rho$ on $\sigma$ operates less effectively, as $N_{s}$ increases at each $Q$ value, or as $Q$ becomes larger with $N_{s}$ fixed.

(ii) The mode energy becomes very close to $\hbar \omega_{\mathrm{LO}}$ in a small $Q$ region at $N_{s}=0 \mathrm{~cm}^{-2}$. The decline in the screening effect in (i) leads to a gradual downward shift in the mode energy toward $\hbar \omega_{\mathrm{SPH}}$, namely, the surface optical-phonon energy in the absence of carriers.

(iii) The decline in the screening effect in (i) entails a remarkable increase in the surface energy-loss intensity.

(iv) From an experimental standpoint, the downward shift in the mode energy in (ii) is too small to observe. The virtu- 
ally undetectable shift in the mode energy in (ii) and the noticeable increase in the loss intensity in (iii) account for the experimental result that the middle loss peak in the EEL spectrum grows conspicuously without any substantial shift, with an increase in $N_{s}$, or with a decrease in the incidentelectron energy.

(v) Regardless of $N_{s}$, contour lines of $\delta \rho(x, z)$ exhibit outstanding tails streaming in the opposite direction to $\mathbf{Q}$. This implies that the surface optical phonon propagates along the surface dragging the screening charges $\delta \rho$.

(vi) As $N_{s}$ increases with $Q$ fixed, the screening charges $\delta \rho$ begin to be distributed in a thicker layer, as is consistent with the fact that $n$ rises from zero more slowly near the surface and with the decline in the screening effect. (vii) As $Q$ becomes larger with $N_{s}$ fixed, the screening charges $\delta \rho$ start to extend into the deeper region, and the dragging character in (v) begins to decline, as is consistent with the attenuation in the screening effect.

\section{ACKNOWLEDGMENTS}

This work is supported by a Grant-in-Aid for Scientific Research from the Ministry of Education, Science, Sport, and Culture under Grant No. 09640386. Numerical calculations in this work were performed at the Tohoku University Computer Center and the Iwate University Computer Center.
${ }^{1}$ C. B. Duke, S. L. Richardson, A. Paton, and A. Kahn, Surf. Sci. 127, L135 (1983).

${ }^{2}$ S. Y. Tong, W. N. Mei, and G. Xu, J. Vac. Sci. Technol. B 2, 393 (1984).

${ }^{3}$ L. Smith, T. E. Derry, and J. F. van der Veen, Surf. Sci. 150, 245 (1985).

${ }^{4}$ R. M. Feenstra, J. A. Stroscio, J. Tersoff, and A. P. Fein, Phys. Rev. Lett. 58, 1192 (1987).

${ }^{5}$ J. E. Gayone, R. G. Pregliasco, G. R. Gómez, E. A. Sánchez, and O. Grizzi, Phys. Rev. B 56, 4186 (1997).

${ }^{6}$ C. Mailhiot, C. B. Duke, and D. J. Chadi, Surf. Sci. 149, 366 (1985).

${ }^{7}$ A. C. Ferraz and G. P. Srivastava, Surf. Sci. 182, 161 (1987).

${ }^{8}$ G.-X. Qian, R. M. Martin, and D. J. Chadi, Phys. Rev. B 37, 1303 (1988).

${ }^{9}$ J. L. A. Alves, J. Hebenstreit, and M. Scheffler, Phys. Rev. B 44, 6188 (1991).

${ }^{10}$ D. J. Chadi, J. Vac. Sci. Technol. A 5, 834 (1987).

${ }^{11}$ M. D. Pashley, Phys. Rev. B 40, 10481 (1989), and references therein.

${ }^{12}$ F. Bartels, L. Surkamp, H. J. Clemens, and W. Mönch, J. Vac. Sci. Technol. B 1, 756 (1983).

${ }^{13}$ C. Astaldi, L. Sorba, C. Rinaldi, R. Mercuri, S. Nannarone, and C. Calandra, Surf. Sci. 162, 39 (1985).

${ }^{14}$ O. M'Hamedi, F. Proix, and C. Sébenne, Semicond. Sci. Technol. 2, 418 (1987).

${ }^{15}$ T. U. Kampen, L. Koenders, K. Smit, M. Rückschloss, and W. Mönch, Surf. Sci. 242, 314 (1991).

${ }^{16}$ A. Ruocco, S. Nannarone, M. Sauvage-Simkin, N. Jedrecy, R. Pinchaux, and A. Waldhauer, Surf. Sci. 307-309, 662 (1994).

${ }^{17}$ A. Ruocco, M. Biagini, A. di Bona, N. Gambacorti, S. Valeri, S. Nannarone, A. Santoni, and J. Bonnet, Phys. Rev. B 51, 2399 (1995).

${ }^{18}$ J. E. Gayone, R. G. Pregliasco, E. A. Sánchez, and O. Grizzi, Phys. Rev. B 56, 4194 (1997).

${ }^{19}$ F. Manghi, C. M. Bertoni, C. Calandra, and E. Molinari, J. Vac. Sci. Technol. 21, 371 (1982).

${ }^{20}$ P. Jiang, Z. Yang, and F. Chen, Chin. Phys. 4, 985 (1984).

${ }^{21}$ C. M. Bertoni, M. Buongiorno Nardelli, F. Bernardini, F. Finoc- chi, and E. Molinari, Europhys. Lett. 13, 653 (1990).

${ }^{22}$ A. F. Wright, C. Y. Fong, and I. P. Batra, Surf. Sci. 244, 51 (1991).

${ }^{23}$ R. Di Felice, A. I. Shkrebtii, F. Finocchi, C. M. Bertoni, and G. Onida, J. Electron Spectrosc. Relat. Phenom. 64/65, 697 (1993).

${ }^{24}$ J. Fritsch, A. Eckert, P. Pavone, and U. Schröder, J. Phys.: Condens. Matter 7, 7717 (1995).

${ }^{25}$ H. Lüth and R. Matz, Phys. Rev. Lett. 46, 1652 (1981).

${ }^{26}$ U. del Pennino, C. Mariani, A. Amoddeo, R. Biagi, F. Proix, and C. Sébenne, J. Phys.: Condens. Matter 5, 6613 (1993).

${ }^{27}$ Z. J. Gray-Grychowski, R. G. Egdell, B. A. Joyce, R. A. Stradling, and K. Woodbridge, Surf. Sci. 186, 482 (1987).

${ }^{28}$ M. Noguchi, K. Hirakawa, and T. Ikoma, Surf. Sci. 271, 260 (1992).

${ }^{29}$ T. S. Jones, M. O. Schweitzer, N. V. Richardson, G. R. Bell, and C. F. McConville, Phys. Rev. B 51, 17675 (1995).

${ }^{30}$ G. R. Bell, C. F. McConville, C. P. A. Mulcahy, and T. S. Jones, J. Phys.: Condens. Matter 9, 2903 (1997).

${ }^{31}$ G. A. Baraff and J. A. Appelbaum, Phys. Rev. B 5, 475 (1972).

${ }^{32}$ D. H. Ehlers and D. L. Mills, Phys. Rev. B 34, 3939 (1986).

${ }^{33}$ S. R. Streight and D. L. Mills, Phys. Rev. B 37, 965 (1988).

${ }^{34}$ H. Yu and J. C. Hermanson, Phys. Rev. B 40, 11851 (1989).

${ }^{35}$ Y. Chen, S. Nannarone, J. Schaefer, J. C. Hermanson, and G. J. Lapeyre, Phys. Rev. B 39, 7653 (1989).

${ }^{36}$ T. Inaoka, Surf. Sci. 431, 156 (1999).

${ }^{37}$ R. Matz and H. Lüth, Phys. Rev. Lett. 46, 500 (1981).

${ }^{38} \mathrm{Ph}$. Lambin, J. P. Vigneron, and A. A. Lucas, Phys. Rev. B 32, 8203 (1985).

${ }^{39}$ T. Inaoka and T. Chihara, Surf. Sci. 208, 71 (1989).

${ }^{40}$ W. L. Schaich, Surf. Sci. 122, 175 (1982).

${ }^{41}$ D. H. Ehlers and D. L. Mills, Phys. Rev. B 36, 1051 (1987).

${ }^{42}$ S. R. Streight and D. L. Mills, Phys. Rev. B 38, 8526 (1988).

${ }^{43}$ S. R. Streight and D. L. Mills, Phys. Rev. B 40, 10488 (1989).

${ }^{44}$ T. Inaoka, Surf. Sci. 257, 237 (1991).

${ }^{45}$ T. Inaoka, Surf. Sci. 351, 259 (1996).

${ }^{46}$ T. Inaoka, Appl. Surf. Sci. 169/170, 51 (2001).

${ }^{47}$ T. Ando, Z. Phys. B 26, 263 (1977).

${ }^{48}$ R. G. Dandrea, N. W. Ashcroft, and A. E. Carlsson, Phys. Rev. B 34, 2097 (1986). 
${ }^{49}$ J. P. Perdew and Y. Wang, Phys. Rev. B 45, 13244 (1992).

${ }^{50}$ J. P. Perdew and A. Zunger, Phys. Rev. B 23, 5048 (1981).

${ }^{51}$ D. M. Newns, Phys. Rev. B 1, 3304 (1970).

${ }^{52}$ T. Inaoka, Surf. Sci. 198, 491 (1988).

${ }^{53}$ J. Lindhard, K. Dan. Vidensk. Selsk. Mat. Fys. Medd. 28, 8 (1954).
${ }^{54}$ A. Raymond, J. L. Robert, and C. Bernard, J. Phys. C 12, 2289 (1979).

${ }^{55}$ E. O. Kane, J. Phys. Chem. Solids 1, 249 (1957).

${ }^{56}$ J. S. Blakemore, J. Appl. Phys. 53, R123 (1982).

${ }^{57}$ T. Inaoka, J. Phys.: Condens. Matter 3, 4825 (1991).

${ }^{58}$ A. Stahl, Surf. Sci. 134, 297 (1983). 